\title{
Untangling Basal Ganglia Network Dynamics and Function: Role of Dopamine Depletion and Inhibition Investigated in a Spiking Network Model
}

\author{
Mikael Lindahl, ${ }^{1,3}$ and (1)Jeanette Hellgren Kotaleski ${ }^{1,2,3}$
}

DOI:http://dx.doi.org/10.1523/ENEURO.0156-16.2016

${ }^{1}$ Science for Life Laboratory, School of Computer Science and Communication, KTH Royal Institute of Technology, Box 1031, 17121 Solna, Sweden, '2Department of Neuroscience, Karolinska Institute, 17177 Stockholm, Sweden, and ${ }^{3}$ Stockholm Brain Institute, Karolinska Institute, 17177 Stockholm, Sweden

\begin{abstract}
The basal ganglia are a crucial brain system for behavioral selection, and their function is disturbed in Parkinson's disease (PD), where neurons exhibit inappropriate synchronization and oscillations. We present a spiking neural model of basal ganglia including plausible details on synaptic dynamics, connectivity patterns, neuron behavior, and dopamine effects. Recordings of neuronal activity in the subthalamic nucleus and Type A (TA; arkypallidal) and Type I (TI; prototypical) neurons in globus pallidus externa were used to validate the model. Simulation experiments predict that both local inhibition in striatum and the existence of an indirect pathway are important for basal ganglia to function properly over a large range of cortical drives. The dopamine depletion-induced increase of AMPA efficacy in corticostriatal synapses to medium spiny neurons (MSNs) with dopamine receptor D2 synapses (CTX-MSN D2) and the reduction of MSN lateral connectivity (MSN-MSN) were found to contribute significantly to the enhanced synchrony and oscillations seen in PD. Additionally, reversing the dopamine depletion-induced changes to CTX-MSN D1, CTX-MSN D2, TA-MSN, and MSN-MSN couplings could improve or restore basal ganglia action selection ability. In summary, we found multiple changes of parameters for synaptic efficacy and neural excitability that could improve action selection ability and at the same time reduce oscillations. Identification of such targets could potentially generate ideas for treatments of PD and increase our understanding of the relation between network dynamics and network function.
\end{abstract}

Key words: basal ganglia; dopamine; inhibition; network dynamics; Parkinson's disease; spiking network model

\section{Significance Statement}

Basal ganglia (BG) are important for selection of behavior, and in Parkinson's disease (PD), dopamine deficiency causes BG to malfunction. Also, the network dynamic behavior changes, and oscillations and spike synchronization develop. We built a BG network model and used it to better understand how network parameters contribute to function as well as network dynamics, and how functionality can be recovered in the disease state. Our findings improve the general understanding of how BG function and which network parameters are associated with impaired function versus when disease-associated parameter changes can be seen as compensatory. Our results may contribute to novel approaches for the treatment of PD.

\section{Introduction}

Basal ganglia (BG) are critical for the initiation and selection of behaviors and actions, and Parkinson's dis-

Received June 10, 2016; accepted November 27, 2016; First published December 22, 2016. ease $(\mathrm{PD})$ caused by dopamine depletion can be linked to inappropriate neural activity in the BG together with im-

Authors report no conflict of interest.

Author Contributions: ML and JHK designed research, ML performed research, ML analyzed data, ML and JHK wrote the paper. 
A

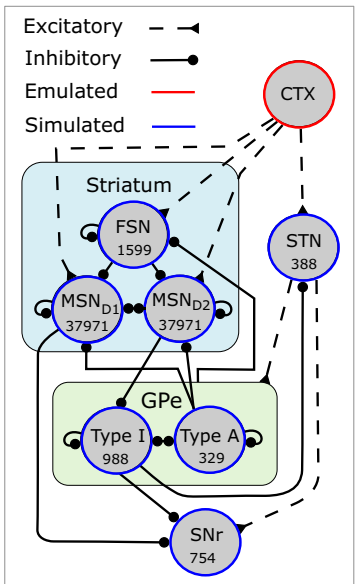

B

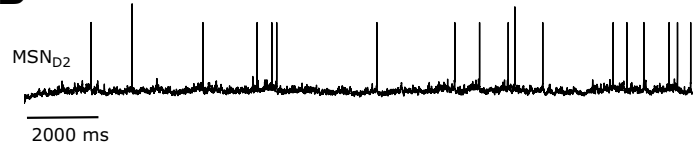

FSN

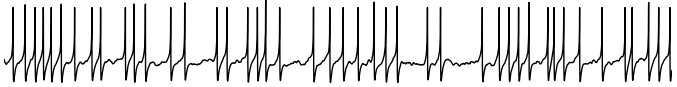
STN

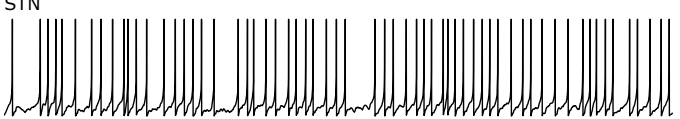

GPe Type I

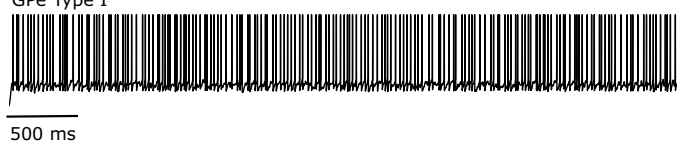

C

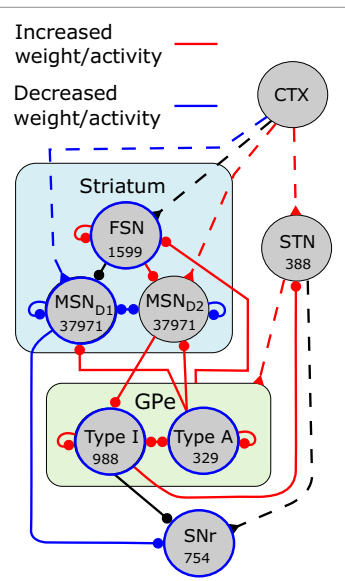

Figure 1. Model description. A, Illustration of the structures included and number of neurons in each simulated nucleus (blue). Solid black lines represent inhibitory synaptic connections; dashed lines represent excitatory synaptic connections. Cortical input was emulated (red). B, Example traces of simulated MSN D2, FSN, STN, and GPe TI neuronal activity. C, Illustration of dopamine-depletion effects on neurons and connections in the network model.

paired function. To improve treatments of PD, we need to understand the underlying neural mechanisms causing $B G$ to malfunction. The effect of dopamine depletion on individual BG network components has been thoroughly studied; however, we still lack an understanding of how the combined effects of altered synaptic efficacy, connectivity, and neural excitability are responsible for the BG dysfunction in PD.

$B G$ have been hypothesized to act as a general action selection device resolving conflicts between potential actions/behaviors competing for restricted resources (Houk and Beiser, 1998; Redgrave et al., 1999; Frank, 2005; Kamali Sarvestani et al., 2011), and in line with this, it has been shown that stimulation of striatum, the main input nucleus of BG, can either promote or inhibit actions (Kravitz et al., 2010; Freeze et al., 2013). Dopamine loss underlying PD (Hornykiewicz, 1966) causes alteration in many places in BG (Cepeda et al., 1993; Shen and Johnson, 2000; Bracci et al., 2002; Hernández-Echeagaray et al., 2004; Hernández et al., 2006; Baufreton and Bevan, 2008; Taverna et al., 2008; Zhou et al., 2009; Humphries et al., 2009a; Chan et al., 2011; Chuhma et al., 2011; Gittis et al., 2011; Miguelez et al., 2012). Computational modeling studies have given us valuable insights into the neural mechanisms behind PD (Terman et al., 2002; Humphries

European Union Seventh Framework Programme (FP7/2007-2013) under grant agreement $n^{\circ} 604102$ (HBP), The European Horizon2020 Framework Programme under grant agreement $n^{\circ} 720270$ (Human Brain Project SGA1), the Swedish Research Council, NIAAA (grant 2R01AA016022), Swedish e-Science Research Center and Stockholm Brain Institute.

Correspondence should be addressed to Mikael Lindahl, Science for Life Laboratory, School of Computer Science and Communication, KTH Royal Institute of Technology, Box 1031, 17121 Solna, Sweden. E-mail: lindahlm@csc.kth.se.

DOI:http://dx.doi.org/10.1523/ENEURO.0156-16.2016

Copyright (c) 2016 Lindahl and Hellgren Kotaleski

This is an open-access article distributed under the terms of the Creative Commons Attribution 4.0 International, which permits unrestricted use, distribution and reproduction in any medium provided that the original work is properly attributed. et al., 2006; Kumar et al., 2011; Damodaran et al., 2014, 2015; Corbit et al., 2016), but typically incorporated a subset of the alterations that dopamine depletion causes and investigated the effects in a subset of BG network components. Thus, to get a further understanding of the neural mechanisms in PD, we aimed to study them in a larger network context and try to relate function and dynamic features seen in experiments.

Focal microinjections of the GABA-A antagonist bicuculline in the striatum lead to loss of specificity (LOS) in BG firing patterns (Bronfeld and Bar-Gad, 2011) and cause repetitive motor tics confined to a single or a few muscles (McCairn et al., 2009). Bronfeld and Bar-Gad (2011) showed that LOS actually is a general phenomenon in BG movement disorders, including PD. The activity of the projection neurons $(\sim 95 \%)$ in the striatum, the medium spiny neuron (MSN), is controlled by, e.g., recurrent inhibition and feed-forward inhibition from fast spiking neurons (FSNs). Recently, another major source of inhibition from globus pallidus externa (GPe) Type A (TA; arkypallidal) was confirmed (Mallet et al., 2012). The effect of this new pathway on striatal activity has not been studied much in computational models. Thus, to get a better understanding of how the different inhibitory inputs that MSNs receive relate to LOS, there is a need to build a model that accounts for this new pathway.

Here we present a quantitative computational model of the BG (Fig. 1). The model includes the striatal network with feedback inhibition from MSNs, feed-forward inhibition from FSNs, and pallidal inhibition from GPe TA neurons, the subthalamic nucleus (STN)-GPe pathway, and the output nucleus substantia nigra reticulata (SNr). Where appropriate, short-term synaptic plasticity is represented. The purpose of building this model was to further test the action selection hypothesis, better understand the underlying neural mechanism of synchrony and oscillations seen in PD, and possibly identify novel targets for treating basal ganglia diseases such as PD. We thus 
investigate and show how BG network components may support action selection, and how intrastriatal inhibition and alterations in the BG nuclei seen in PD can influence BG network dynamics. Inhibition of MSNs by FSNs and $\mathrm{GPe}$ complement the inhibition from MSN collaterals, where the former are most effective during low cortical activity and the latter at higher cortical drive. We predict that the weak but numerous MSN collaterals have a sufficiently strong effect so that active populations of MSNs can significantly suppress the activity of neighboring neurons even when only a small striatal MSN population is bursting after local activation. We find in our model that the strengthening of the drive from cortex to MSN D2, the weakening of the MSN collaterals, and the altered properties of the GPe network, which all are changes associated with dopamine depletion, are very important BG network alterations behind the increased network synchrony and oscillations seen in PD. We see that intrastriatal inhibition can regulate and improve action selection contrast over a range of input strengths, and the activation of STN can delay or prevent action selection in the control network and also improve function in the dopamine-depleted network. Finally, we search for network perturbations of synaptic efficacy and neural excitability that could improve action selection capability or decrease oscillations in PD.

\section{Materials and Methods}

\section{Network model}

The model network consists of a population of MSN D1, MSN D2, FSN, STN, GPe TA, GPe Type I (TI), and SNr neurons modeled as point neurons (Fig. 1A; Mallet et al., 2008). In this study, striatum consisting of MSN D1, MSN D2, and FSNs as well as GPe TA and TI neuron populations (Mallet et al., 2008) were added to a previously published model (Lindahl et al., 2013) in which only the STN, GPe, and SNr network was modeled. Furthermore, we have added known dopamine modulation to neuron types and synapses (Cepeda et al., 1993; Shen and Johnson, 2000; Bracci et al., 2002; Hernández-Echeagaray et al., 2004; Hernández et al., 2006; Baufreton and Bevan, 2008; Taverna et al., 2008; Zhou et al., 2009; Humphries et al., 2009a; Chan et al., 2011; Chuhma et al., 2011; Gittis et al., 2011; Miguelez et al., 2012). All populations received external excitatory uncorrelated Poisson synaptic input to achieve realistic baseline firing rates. Here, cortex would represent the main source of the excitatory input for MSNs, FSNs, and STN, whereas thalamus and brainstem nuclei could be the source of the excitatory input to GPe and SNr (Smith et al., 2010).

A basal ganglia network size of 80,000 neurons was used for all simulations except in simulations in which we study action selection with a network size of 20,000 and when studying inhibition in a striatal network module of $\sim 3000$ neurons. The numbers of neurons used for each nucleus in the model with 80,000 neurons are displayed in Table 1 (see also Fig. 1A). The sizes of the nuclei were calculated by using the relative proportion of $B G$ nuclei obtained from the study of absolute numbers of neurons in striatum, STN, GPe, and SNr in rat (Oorschot, 1996) and together with information about the relative distribution of MSN D1, MSN D2 and FSN in striatum [47.5\%, 47.5\%, and 2\%, respectively (Gerfen et al., 2010; Tepper, 2010)] and the relative distribution of GPe TA (arkypallidal) and GPe TI (prototypical) [25\% and 75\% (Abdi et al., 2015)]. A subpopulation of GPe $\mathrm{TI}$ neurons have been shown to project back to FSN (Abdi et al., 2015; Saunders et al., 2016), which we estimated as $10 \%$ of the total TI population. With a neural density of $84,900 \mathrm{~mm}^{-3}$ (Oorschot, 1996) in striatum, $95 \%$ of which are estimated to be MSNs, and radii of the axonal and dendritic arborizations both $\sim 200 \mu \mathrm{m}$ (Gerfen et al., 2010), we estimate that there are $2800 \mathrm{MSNs}$ within the volume of the axonal field of one MSN $(89,400 \times 0.95 \times 0.0335)$. With a volume of 6.5 $\times 10^{-3} \mathrm{~mm}$ (Tepper, 2010) of the axonal arborization of FSNs, we estimate that $540(89,400 \times 0.95 \times 0.0065)$ MSNs are within the axonal field of an FSN. Spatial restrictions were applied only to MSN collateral connectivity and FSN-to-MSN connectivity, based on the data on dendritic and axonal innervation stated above, such that an MSN or FSN could contact only $2800 / 540$ other MSNs assumed to be in closest proximity. All other synaptic connections between and within different nuclei were randomly distributed, since axons and dendritic trees generally project over large target areas in BG (Smith et al., 1998; Sadek et al., 2007; Baufreton et al., 2009; Mallet et al., 2012).

To introduce variations into the neural populations, the capacitance $(C)$ and spike threshold $\left(V_{T} / V_{t h}\right)$ were assumed to be Gaussian distributed around a mean capacitance, $C$, and spike threshold, $V_{T} / V_{t h}$, respectively. As for the standard deviation of the capacitance, we assumed it to be $10 \%$ of the mean, and for the spike threshold, the deviation was set to be $1 \mathrm{mV}$.

\section{Neuronal firing rates}

To ensure realistic population firing rates, we adjusted the Poisson input to each population $v$ (Table 1, network and connection parameters). The activity of GPe TA, GPe TI, and STN neurons were matched to Mallet et al. (2008). As for the other populations, it was ensured that their basal firing rates were in the range of values recorded in in vivo experiments, e.g., MSN $0.01-2.0 \mathrm{~Hz}$ (Miller et al., 2008), FSN 10-20 Hz (Berke et al., 2004; Gage et al., 2010), and SNr 20-35 Hz (Maurice et al., 2003; Gernert et al., 2004; Zahr et al., 2004; Walters et al., 2007).

\section{Modeling extrinsic inputs to the basal ganglia network}

Input to MSNs, FSNs, STN, GPe populations, and SNr were modeled in all simulations as Poisson processes with the frequencies $v$ listed in Table 1 for the states denoted, respectively, cortical activation and cortical slow-wave activity. Cortical activation represents the activity following a hindpaw pinch (under urethane anesthesia), whereas slow-wave activity represents the activity at rest (also under urethane anesthesia; Mallet et al., 2008). A $1-\mathrm{Hz}$ frequency modulation (used during both control and dopamine-depletion experiments) was used for generating the cortical slow-wave activity, and a $20-\mathrm{Hz}$ modulation was used to generate beta oscillations (used 
Table 1. Network and connection parameters

\begin{tabular}{|c|c|c|}
\hline Name & Value & Description \\
\hline$N_{\text {network }}$ & 80,000 & Network size \\
\hline$N_{\text {network }}^{M S N_{D 1}}$ & 37,971 & Size of MSN D1 population \\
\hline$N_{\text {network }}^{M S N_{D 2}}$ & 37,971 & Size of MSN D2 population \\
\hline$N_{\text {network }}^{F S N}$ & 1599 & Size of FSN population \\
\hline$N_{\text {network }}^{\text {STN }}$ & 388 & Size of STN population \\
\hline$N_{\text {network }}^{G P e_{\text {TA }}}$ & 329 & Size of GPe TA population \\
\hline$N_{\text {network }}^{G P e_{\text {TI }}}$ & 988 & Size of GPe TI population \\
\hline$N_{\text {network }}^{S N r}$ & 754 & Size of SNr population \\
\hline$V^{C T X-M S N_{D 1}}$ & $448 / 546 \mathrm{~Hz}$ & Cortical input to MSN D1 (slow-wave/activation) \\
\hline$V^{C T X-M S N_{D 2}}$ & $592 / 722 \mathrm{~Hz}$ & Cortical input to MSN D2 (slow-wave/activation) \\
\hline$V^{C T X-F S N}$ & $646 / 787 \mathrm{~Hz}$ & Cortical input to FSN (slow-wave/activation) \\
\hline$v^{\text {CTX-STN }}$ & $170 / 250 \mathrm{~Hz}$ & Cortical input to in STN (slow-wave/activation) \\
\hline$V^{E X T-G P e_{T A}}$ & $100 / 200 \mathrm{~Hz}$ & External input to GPe TA (slow-wave/activation) \\
\hline$V^{E X T-G P e_{T I}}$ & $720 / 1530 \mathrm{~Hz}$ & External input to GPe TI (slow-wave/activation) \\
\hline$v^{E X T-S N r}$ & $1800 / 1800 \mathrm{~Hz}$ & External input to SNr (slow-wave/activation) \\
\hline$a^{C T X-M S N_{D 1}}$ & 0.11 & Amplitude oscillations MSN D1 (both states) \\
\hline$a^{C T X-M S N_{D 2}}$ & 0.11 & Amplitude oscillations MSN D2 (both states) \\
\hline$a^{C T X-F S N}$ & 0.11 & Amplitude oscillations FSN (both states) \\
\hline$a^{C T X-S T N}$ & $0.11 / 0.35$ & Amplitude oscillations STN (slow-wave/activation) \\
\hline 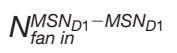 & 364 & Number of MSN D1 connections on each MSN D1 \\
\hline 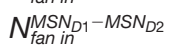 & 84 & Number of MSN D1 connections on each MSN D2 \\
\hline$N_{\text {fan in }}^{M S N_{D 2}-M S N_{D 1}}$ & 392 & Number of MSN D2 connections on each MSN D1 \\
\hline$N_{\text {fan in }}^{M S N_{D 2}-M S N_{D 2}}$ & 504 & Number of MSN D2 connections on each MSN D2 \\
\hline$N_{\text {fan in }}^{F S N-M S N_{D 1}}$ & 16 & Number of FSN connections on each MSN neuron \\
\hline$N_{\text {fan in }}^{F S N-M S N_{D 2}}$ & 11 & Number of FSN connections on each MSN neuron \\
\hline$N_{\tan }^{G P e_{T A}}-M S N$ & 10 & Number of GPe TA connections on each MSN neuron \\
\hline$N_{\text {fan in }}^{F S N-F S N}$ & 10 & Number of FSN connections on each FSN neuron \\
\hline$N_{\text {fan in }}^{G P e_{T A}-F S N}$ & 10 & Number of GPe TA connections on each FSN neuron \\
\hline$N_{\text {fan in }}^{G P e_{\text {TI }}-F S N}$ & 10 & Number of GPe TI connections on each FSN neuron \\
\hline$N_{\text {fan in }}^{G P e_{T / 1}-S N r}$ & 32 & Number of GPe connections on each SNr neuron \\
\hline 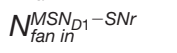 & 500 & Number of MSN D1 connections on each SNr neuron \\
\hline$N_{\text {fan in }}^{S T N-S N r}$ & 30 & Number of STN connections on each SNr neuron \\
\hline$N_{\text {fan in }}^{M S N_{D 2}-G P e_{T I}}$ & 500 & Number of MSN D2 connections on each GPe TI neuron \\
\hline$N_{\text {fan in }}^{S T N-G P e}$ & 30 & Number of STN connections on each GPe neuron \\
\hline$N_{\text {fan in }}^{G P e_{T A}-G P e_{T A}}$ & 5 & Number of GPe TA reciprocal connections \\
\hline$N_{\text {fan in }}^{G P e_{A}}-G P e_{T l}$ & 5 & Number of GPe TA connections on each GPe TI \\
\hline$N_{\text {fan in }}^{G P}-G P e_{T A}$ & 25 & Number of GPe TI connections on each GPe TA \\
\hline$N_{\text {fan in }}^{G P e_{T H}-G P e_{T l}}$ & 25 & Number of GPe TI reciprocal connections \\
\hline$N_{\text {fan in }}^{G P e_{T}}-S T N$ & 30 & Number of GPe TI connections on each STN neuron \\
\hline
\end{tabular}

during dopamine-depletion experiments) during cortical activation. These frequency-modulated inputs were simulated as \pm changes in Poisson frequency with a factor a (Table 1) such that the instantaneous frequency was changed between $v+v * a$ and $v-v * a$ every half cycle of the modulatory input.

For simulations in which we tested action selection capability of the model, the input was modeled as a transient increase in the Poisson inputs to MSNs, FSNs, and STN neurons. The characteristics of MSN activity in vivo (in both anesthetized and nonanesthetized preparations) is a low-frequency firing interrupted by bursts (Wilson, 1993). The basal firing rate for MSNs ranged in simulations between 0.01 and $2.0 \mathrm{~Hz}$, whereas spike frequency during the bursts ranged between 17 and $48 \mathrm{~Hz}$ (Miller et al., 2008). It has been suggested that dynamic synapses (which are included in the current model) modify action selection outcome differentially at different burst lengths (Lindahl et al., 2013); thus, to reduce the complexity of the interpretation of results, we set the burst length to $100 \mathrm{~ms}$, in line with experiments showing that MSNs usually burst for at least $100 \mathrm{~ms}$ (and sometimes up to 1 s; Miller et al., 2008; Gage et al., 2010). This was done in all simulations testing action selection in the model. Action selection simulations consisted of multiple trials, in which each trial consisted of a selection phase with a $100-m s$ burst in specific MSN populations followed by a 900-ms rest phase with only background input drive to MSNs. For each input combination of two hypothesized competing actions, we ran 80 trials to account for trial variability. To generate a full plot with, e.g., $7 \times 7$ cortical input combinations (where typically one input was stron- 
Table 2. FSN model parameters (quadratic integrate and fire model)

\begin{tabular}{|c|c|c|}
\hline Name & Value & Description \\
\hline a & $0.2 \mathrm{~s}$ & Recovery current time constant \\
\hline$b$ & 0.025 & Voltage dependency of recovery current \\
\hline c & $-60 \mathrm{mV}$ & Spike reset \\
\hline C & 80 pF & Membrane capacitance \\
\hline$d$ & $0 \mathrm{pA}$ & $\begin{array}{l}\text { Summed recovery current contribution } \\
\text { following an action potential }\end{array}$ \\
\hline$k$ & 1 & Steady-state voltage dependence \\
\hline$\beta_{v_{r}}$ & -0.078 & $\begin{array}{l}\text { Magnitude of dopamine effect on resting } \\
\text { potential } v_{t}\end{array}$ \\
\hline$v_{b}$ & $-55 \mathrm{mV}$ & Voltage dependence recovery current \\
\hline$v_{\text {peak }}$ & $25 \mathrm{mV}$ & Spike cutoff \\
\hline$v_{r}$ & $-64.4 \mathrm{mV}$ & Resting potential \\
\hline$v_{\text {th }}$ & $-50 \mathrm{mV}$ & Threshold potential \\
\hline
\end{tabular}

ger than the other), we had to run 3920 selection trials. For each of the $7 \times 7$ (49) combinations of competing action pairs, the result was displayed using a pie chart plot (see below). During action selection simulations with an assumed dopamine depletion, we had cortical beta modulation turned on (see above). Threshold passing during an action selection trial was said to have occurred if the mean firing rate of corresponding $\mathrm{SNr}$ neurons during the selection phase dropped below $50 \%$ of their mean activity in the control model. Thus each selection trial could have four outcomes, and the relative proportion of those were represented in each pie in the pie chart plot: (1) only action 1 was selected; (2) only action 2 was selected; (3) both actions were selected; or (4) neither of the actions was selected.

In simulations in which we tested the effect of striatal inhibition, the input was modeled as a stepwise increase in the amplitude of the simulated cortical input to MSNs, FSNs, and STN.

\section{Point neuron models used}

We used two types of neural models, both hybrid spiking, with one fast and one slow state variable. For FSNs and MSNs, we used the quadratic integrate and fire model with adaptation (Eq. 1; Izhikevich, 2007) and parameters taken from Humphries et al. (2009a,b) (Tables 2 and 4). For STN, GPe, and SNr neurons, we used the adaptive exponential integrate and fire model (Eq 2; Brette and Gerstner, 2005) with parameters taken from Lindahl et al. (2013) (Tables 3, 5 and 6). Both neuron models have a good trade-off between simulation efficacy and ability to capture important dynamic behaviors of neurons (Izhikevich, 2010).

The following equations control the dynamics of the quadratic integrate-and-fire model with adaptation, where $V$ is the membrane potential and $u$ is the contribution of the neuron's slow currents:

$$
\begin{gathered}
C \frac{d V}{d t}=k\left(V-v_{r}\right)\left(V-v_{\mathrm{th}}\right)-u+I, \\
\frac{d u}{d t}=a\left(b\left(V-v_{r}\right)-u\right),
\end{gathered}
$$

Table 3. GPe neuron parameters (adaptive exponential inte-

\begin{tabular}{|c|c|c|}
\hline Name & Value & Description \\
\hline a & $2.5 \mathrm{nS}$ & Subthreshold adaptation \\
\hline$b^{T I}$ & $70 \mathrm{pA}$ & Spike-triggered adaptation TI neurons \\
\hline$b^{T A}$ & $105 \mathrm{pA}$ & Spike-triggered adaptation TA neurons \\
\hline$\beta_{E_{L}}$ & -0.181 & $\begin{array}{l}\text { Magnitude of D1 effect on resting } \\
\text { potential } E_{l}\end{array}$ \\
\hline$C^{T I}$ & $40 \mathrm{pF}$ & Membrane capacitance TI neurons \\
\hline$C^{T A}$ & $60 \mathrm{pF}$ & Membrane capacitance TA neurons \\
\hline$\Delta_{T}^{T \prime}$ & $1.7 \mathrm{~ms}$ & Slope factor of spike upstroke TI neurons \\
\hline$\Delta_{T}^{T A}$ & $2.55 \mathrm{~ms}$ & Slope factor of spike upstroke TA neurons \\
\hline$E_{L}$ & $-55.1 \mathrm{mV}$ & Leak reversal potential \\
\hline$g_{L}$ & $1 \mathrm{nS}$ & Leak conductance \\
\hline$l_{e-T A}$ & $1 \mathrm{pA}$ & $\begin{array}{l}I_{\text {inj }} \text { to obtain in vitro firing rate } 8 \mathrm{~Hz} \text { without } \\
\text { synaptic input }\end{array}$ \\
\hline$I_{e-T I}$ & $12 \mathrm{pA}$ & $\begin{array}{l}I_{\text {inj }} \text { to obtain in vitro firing rate } 18 \mathrm{~Hz} \text { without } \\
\text { synaptic input }\end{array}$ \\
\hline$\tau_{w}$ & $20 \mathrm{~ms}$ & Adaptation time constant \\
\hline$t^{f}$ & $15 \mathrm{mV}$ & Spike cut off \\
\hline$V_{r}$ & $-60 \mathrm{mV}$ & Spike reset \\
\hline$V_{T}$ & $-54.7 \mathrm{mV}$ & Threshold potential \\
\hline
\end{tabular}
grate and fire model)

$$
\text { if } V>v_{\text {peak }} \text { then } v=c \text { and } u=u+d \text {. }
$$

Here $C$ is the capacitance, $v_{r}$ and $v_{\text {th }}$ are the resting and threshold potentials, $I$ is a current source, $a$ is the recovery current time constant, $b$ is the voltage dependence of the recovery current, and $k$ is a parameter determining the steady-state current voltage $(I-V)$ relation. When the membrane potential $V$ reaches $v_{\text {peak }}$, it is reset to $c$, and then the recovery current $u$ is updated with $d$.

The following equations control the dynamics of the adaptive exponential integrate-and-fire model, where $V$ is the membrane potential and $w$ is the contribution of the neuron's slow currents:

$$
\begin{gathered}
C \frac{d V}{d t}=-g_{L}\left(V-E_{L}\right)+g_{L} \Delta_{T} \exp \left(\frac{V-V_{T}}{\Delta_{T}}\right)-w+I, \\
\tau_{w} \frac{d w}{d t}=a\left(V-E_{L}\right)-w, \\
\text { if } V>t^{f} \text { then } V=V_{r} \text { and } w=w+b .
\end{gathered}
$$

Here $C$ is the capacitance, $g_{L}$ is the leak conductance, $E_{L}$ and $V_{T}$ are the resting and threshold potentials, $\Delta_{T}$ is the slope factor, $I$ is a current source, $\tau_{w}$ is the recovery current time constant, and $a$ is the voltage dependence of the recovery current above. When the membrane potential $V$ reaches $t^{f}$, it is reset to $V_{r}$, and then the recovery current $w$ is increased with $b$.

The effect of tonic dopamine level has been incorporated in the model and is regulated by $\alpha_{d o p}$, a parameter between 0 and 1 representing the relative level of dopamine receptor occupancy, a concept previously used in Humphries et al. (2006, 2009a,b). The dopamine effect on model parameters $p$ is modeled by multiplying them with $1+\beta_{\text {par }} \phi\left(\alpha_{d o p}\right)$ such that $p \leftarrow p\left[1+\beta_{p a r} \phi\left(\alpha_{d o p}\right)\right]$. Here $\beta_{\text {par }} \in[-5,1.25]$ is a scaling coefficient, for correspond- 
ing parameter fitted from experiments, that determines the relationship between dopamine receptor occupancy and the magnitude of the effect. In the function $\phi$ $\left(\alpha_{d o p}\right)=\alpha_{d o p}-\alpha_{0}, \alpha_{0}=0.8$ is considered to be the normal dopamine level in all the simulations.

\section{The FSN model}

The FSN model shows type 2 dynamics with a nonlinear step from silent to spiking, which is modeled by letting $\dot{u}=a\left[b\left(V-v_{b}\right)^{3}-u\right]$ if $v \leq v_{b}$, otherwise $\dot{u}=-a u$ (Izhikevich, 2007; Humphries et al., 2009b). This means that the FSN model can exhibit narrow action potentials with rapid, large, and brief afterhyperpolarizations and display irregular firing around spike threshold (if some noise is added; Tepper, 2010). Dopamine has a depolarizing effect on the FSN through D1 type receptor activation. We model this effect as $v_{r} \leftarrow v_{r}\left(1+\beta_{v} \phi\right)$, where $\beta_{v}$ was set such that the resting potential at low dopamine ( $\left.\alpha_{\text {dop }}=0\right)$ was $5 \mathrm{mV}$ lower than at high dopamine $\left(\alpha_{\text {dop }}=1\right.$; Bracci et al., 2002; Centonze et al., 2003). The model parameters are listed in Table 2.

\section{The GPe neuron model}

The GPe neuron exhibits membrane oscillations close to spike threshold, causing irregular firing and regular firing at higher depolarizing currents (Nambu and Llinaś, 1994; Cooper and Stanford, 2000) as well as a rebound spiking upon release from hyperpolarization (Nambu and Llinaś, 1994; Cooper and Stanford, 2000). It has a linear current-frequency relationship, with strong spike frequency adaptation at higher input (Cooper and Stanford, 2000; Bugaysen et al., 2010). Dopamine has a depolarizing effect on the GPe neurons by up-regulating the $\mathrm{HCN}$ channel responsible for the regular pacemaking of $\mathrm{GPe}$ neurons (Chan et al., 2011). We modeled this effect as $E_{L} \leftarrow E_{L}\left(1+\beta_{E_{L}} \phi\right)$, where $\beta_{E_{L}}$ was tuned such that at low dopamine $\left(\alpha_{\text {dop }}=0\right)$, the resting potential was $10 \mathrm{mV}$ lower than at high dopamine $\left(\alpha_{\text {dop }}=1\right)$.

Abdi et al. (2015) showed that TA neurons have a flatter current-frequency curve (Abdi et al. 2015; 60\% of the TI slope) compared with TI neurons. To account for this, we set the spike triggered adaptation $b$, spike slope factor $\Delta_{T}$, and membrane capacitance $C$ of TA neurons to $150 \%$ of TI neurons (Table 3). For TA and TI neurons to fire at 8 and $18 \mathrm{~Hz}$, respectively, at zero current injection (Abdi et al. 2015), we set $I_{e}^{G P e_{T A}}$ to $1 \mathrm{pA}$ and $l_{e}^{G P e_{T l}}$ to $12 \mathrm{pA}$. In addition to the input from STN, GPe neurons have been shown to receive input from the central medial and parafascicular nucleus of thalamus (Kincaid et al., 1991; Deschênes, 1996; Yasukawa et al., 2004). We include the assumed contribution from these two structures as a Poisson-type external excitatory background input to TA and TI. The GPe neuron model parameters are listed in Table 3.

\section{The MSN models}

The MSN model captures the prominent long latency to spike discharge of MSNs (Nisenbaum et al., 1994) and that MSN D2 cells have a higher input resistance, due to smaller dendritic surface, and are slightly but significantly more excitable than MSN D1 cells (Kreitzer and Malenka, 2007; Gertler et al., 2008). MSN dopamine receptor D1
Table 4. MSN D1 and MSN D2 model parameters (quadratic integrate and fire model)

\begin{tabular}{|c|c|c|}
\hline Name & Value & Description \\
\hline a & $0.01 \mathrm{~s}$ & Recovery current time constant \\
\hline$\alpha$ & 0.032 & Magnitude of D2 effect on $k$ \\
\hline$b$ & -20 & Voltage dependency of recovery current \\
\hline$c$ & $-60 \mathrm{mV}$ & Spike reset \\
\hline C & $15.2 \mathrm{pF}$ & Membrane capacitance \\
\hline$d^{M S N_{D 1}}$ & $66.9 \mathrm{pA}$ & Spike-triggered adaptation MSN D1 \\
\hline$d^{M S N_{D 2}}$ & $91 \mathrm{pA}$ & Spike-triggered adaptation MSN D2 \\
\hline k & 1 & Steady-state voltage dependence \\
\hline$\beta_{v_{r}}^{M S N_{D 1}}$ & 0.0296 & $\begin{array}{l}\text { Magnitude of D1 effect on threshold } \\
\text { potential } v_{t}\end{array}$ \\
\hline$\beta_{d}^{M S N_{D 1}}$ & -0.450 & $\begin{array}{l}\text { Magnitude of D1 effect on recovery } \\
\text { current contribution } d\end{array}$ \\
\hline$v_{\text {peak }}$ & $40 \mathrm{mV}$ & Spike cutoff \\
\hline$V_{r}^{M S N_{D 1}}$ & $-78.2 \mathrm{mV}$ & Resting potential MSN D1 \\
\hline$V_{r}^{M S N_{D 2}}$ & $-80 \mathrm{mV}$ & Resting potential MSN D2 \\
\hline$v_{t h}$ & $-29.7 \mathrm{mV}$ & Threshold potential \\
\hline
\end{tabular}

activation has a hyperpolarizing effect by increased KIR but also enhances the neural response to depolarizing input, see Gruber et al. (2003). The first effect is modeled as a dopamine-dependent change in the threshold poten-

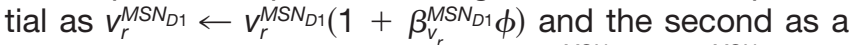
change in the recovery current as $d^{M S N_{D 1}} \leftarrow d^{M S N_{D 1}}(1+$ $\left.\beta_{d}^{M S N_{D 1}} \phi\right)$, and both were set such that the values of $V_{r}$ and $d$ changed with dopamine as in Humphries et al., (2009a). Planert et al. (2013) demonstrate that MSN D2 excitability depends on dopamine concentration; however, interestingly, with low dopamine concentrations $(60 \mu \mathrm{M})$, argued to be more representative for in vivo conditions, no significant (and consistent) change in excitability is seen. Thus we choose to not include any postsynaptic dopamine effect for MSN D2 dopamine receptor activation. The MSN neuron parameters are listed in Table 4.

\section{The SNr neuron model}

The SNr neuron has a linear current frequency relation with spike frequency adaption (Nakanishi et al., 1987; Richards et al., 1997). From holding potential at just below spike threshold, small changes of $\sim 5 \mathrm{pA}$ in injected current are sufficient to bring the neuron from silent to repetitively firing (Atherton and Bevan, 2005), and the SNr neuron exhibits rebound spiking upon release from hyperpolarization (Nakanishi et al., 1987, 1997). Dopamine has a depolarizing effect on the SNr neurons (Zhou et al., 2009). We modeled this effect as $E_{L} \leftarrow E_{L}\left(1+\beta_{E_{L}} \phi\right)$, where $\beta_{E_{L}}$ was set such that at low dopamine $\left(\alpha_{\text {dop }}=0\right)$, the resting potential was $5 \mathrm{mV}$ lower than at high dopamine ( $\left.\alpha_{\text {dop }}=1\right)$. The model parameters are listed in Table 5 .

\section{The STN neuron model}

The STN neuron can fire at high frequency and has a steep current frequency curve (Bevan and Wilson, 1999; Hallworth et al., 2003). Duration of the afterhyperpolarization after a brief depolarization for $\sim 500 \mathrm{~ms}$ should depend on injected current strength (Bevan and Wilson, 1999). Depolarizing the neuron below $-70 \mathrm{mV}$ for a certain period should lead to a rebound burst (Bevan et al., 2000; Hallworth et al., 2003). The hyperpolarization-induced 
Table 5. SNr neuron model parameters (adaptive exponential integrate and fire model)

\begin{tabular}{|c|c|c|}
\hline Name & Value & Description \\
\hline a & $3 \mathrm{nS}$ & Subthreshold adaptation \\
\hline$b$ & 200 pA & Spike-triggered adaptation \\
\hline$\beta_{E_{L}}$ & -0.0896 & $\begin{array}{l}\text { Magnitude of D1 effect on resting } \\
\text { potential } E_{L}\end{array}$ \\
\hline C & 80 pF & Membrane capacitance \\
\hline$\Delta_{T}$ & $1.8 \mathrm{~ms}$ & Slope factor of spike upstroke \\
\hline$E_{L}$ & $-55.8 \mathrm{mV}$ & Leak reversal potential \\
\hline$g_{L}$ & $3 \mathrm{nS}$ & Leak conductance \\
\hline$l_{e}^{L}$ & $15 \mathrm{pA}$ & $\begin{array}{l}I_{i n j} \text { to obtain in vitro firing rate without } \\
\text { synaptic input }\end{array}$ \\
\hline$\tau_{w}$ & $20 \mathrm{~ms}$ & Adaptation time constant \\
\hline$t^{f}$ & $20 \mathrm{mV}$ & Spike cut off \\
\hline$V_{r}$ & $-65 m V$ & Spike reset \\
\hline$V_{T}$ & $-55.2 \mathrm{mV}$ & Threshold potential \\
\hline
\end{tabular}

burst of STN neurons was modeled by resetting $V$ after a spike to $V_{\text {reset }}+\max (w-15,20)$ with $w<0$. The parameters of the STN model are listed in Table 6.

\section{Synaptic connectivity}

Some of the connections (Table 1) in the current model network were obtained from a previously published model (Lindahl et al., 2013). Below we describe the added connections.

For the synapse types MSN D1-MSN D1, MSN D1MSN D2, MSN D2-MSN D1, and MSN D2-MSN D2, the postsynaptic neurons were estimated to receive from neighboring neurons 364, 84, 393, and 504 synapses, respectively. This is motivated in the following way: within the dendritic and axonal tree of an MSN D1 or MSN D2, there are $\sim 2800$ MSNs (see Network model). The connection probability of MSN D1-MSN D1, MSN D1-MSN D2, MSN D2-MSN D1, and MSN D2-MSN D2 is 13\%, $3 \%, 14 \%$, and $18 \%$, respectively (Taverna et al., 2008 ); thus, the respective in-degree adds up to $364,84,393$, and $504(2800 \times 0.13,0.03,0.14$, or 0.18$)$. Similarly, the number of synapses that MSN D1 and MSN D2 neurons receive from FSNs was estimated as 16 and 11, respectively. We have $60 \mathrm{FSNs}$ within the dendritic tree of an MSN assuming $95 \%$ and $2 \%$, respectively, of MSNs and

Table 6. STN neuron parameters (adaptive exponential integrate and fire model)

\begin{tabular}{|c|c|c|}
\hline Name & Value & Description \\
\hline a & $0.3 \mathrm{nS}$ & $\begin{array}{l}\text { Subthreshold adaptation (below }-70 \text { ) } \\
\text { otherwise equal to } 0\end{array}$ \\
\hline$b$ & $0.05 \mathrm{pA}$ & Spike-triggered adaptation \\
\hline C & $60 \mathrm{pF}$ & Membrane capacitance \\
\hline$\Delta_{T}$ & $16.2 \mathrm{~ms}$ & Slope factor of spike upstroke \\
\hline$E_{L}$ & $-80.2 \mathrm{mV}$ & Leak reversal potential \\
\hline$g_{L}$ & $10 \mathrm{nS}$ & Leak conductance \\
\hline$I_{e}$ & $5 \mathrm{pA}$ & $\begin{array}{l}I_{\text {inj }} \text { to obtain in vitro firing rate without } \\
\text { synaptic input }\end{array}$ \\
\hline$\tau_{w}$ & $333 \mathrm{~ms}$ & Adaptation time constant \\
\hline$t^{f}$ & $15 \mathrm{mV}$ & Spike cut off \\
\hline$V_{r}$ & $-70 \mathrm{mV}$ & Spike reset \\
\hline$V_{T}$ & $-64.0 \mathrm{mV}$ & Threshold potential \\
\hline
\end{tabular}

FSNs in striatum (Tepper, 2010; $2800 \div 0.95 \times 0.02$ ). Then with probability of connection at $27 \%$ and $18 \%$ for FSN-MSN D1 and FSN-MSN D2 (Gittis et al., 2010; $60 \times$ 0.27 or 0.18 ), we estimate the number of synapses MSN D1 and MSN D2 neurons receive from FSNs as 16 and 11. FSNs also make synapses onto other FSNs, with $74 \%$ probability of contacting a neighboring FSN (Gittis et al., 2010). Because there are $540 \mathrm{MSN}$ within the axonal field of an FSN, we can estimate the number of FSNs within the axonal field to $12(560 \div 0.95 \times 0.02)$. Thus we estimate that one FSN makes contact with nine other FSNs $(0.74 \times$ 12). The MSNs and FSNs were ensured to compile with spatial aspects of biology in which each MSN and FSN only were considered connecting neighboring neurons up to the numbers that each axonal tree contains (2800 and 540 , respectively).

Kita and Kitai (1994) and Bevan et al. (1998) found by labeling neurons and tracing axons that a population of GPe neurons ( $\sim 25 \%)$ project more or less exclusively to striatum. In Mallet et al. (2008) it was shown, based on the discharge pattern during slow-wave sleep, that there exist two types of GPe neurons: TA, which preferentially discharge during the "active" component of the slow $1-\mathrm{Hz}$ cortical oscillation, and $\mathrm{TI}$, which preferentially discharged during the negative "inactive" component. The study suggested that TA preferentially is driven by excitation from STN and that TI is inhibited from the striatum. Chuhma et al. (2011) supported the claim that there is a population of GPe neurons not receiving much input from MSNs by showing that $28 \%$ of the GPe neurons in their study were not activated by light stimulation of striatal fibers. In recent studies (Mallet et al., 2012, 2016; Gittis et al., 2014; Abdi et al., 2015) it was shown that TA and TI have different structural connectivity in which TA strongly connects to striatum (but not to STN or to basal ganglia output nuclei) and $\mathrm{TI}$ preferably connects to $\mathrm{BG}$ output nuclei (and STN). Abdi et al. (2015) also showed that a subpopulation of Tl cells (estimated to 10\%) indeed projects to striatum. We thus include in our model TA neurons that project to striatum, but not to STN. TI neurons are also included which, in contrast to TA project to STN, receive input from striatum, and where one out of every tenth TI neuron projects back to striatum. Specifically, in the striatum, TA neurons project to both MSNs and FSNs, whereas TI neurons project only to FSNs in accordance with the literature (Glajch et al., 2016; Saunders et al., 2016).

Mallet et al. (2012) established that a TA neuron gives rise to $\sim 10,000$ synaptic boutons in striatum, but the relative innervations of FSNs and MSNs were not determined. There are up to 100 times more MSNs than FSNs in striatum, and therefore we assume that for each connection GPe makes with FSNs, it contacts 100 MSNs. Furthermore, we assumed that 1000 is the maximal number of MSNs that a single TA neuron connects to. Then, on average, each MSN receives input from $10 \mathrm{GPe}(1000$ connections $\div 100=\mathrm{MSN} / \mathrm{TA}$ ratio), and thus each TA cell is assumed to make 10 boutons on each MSN. We then have that $1 \mathrm{TA}$ cell makes 10,000 boutons onto MSNs $(10 \times 1000=10,000$ synaptic boutons $)$. For an 
FSN, we assume that it also receives input from $10 \mathrm{TA}$ and that each TA makes 10 boutons on each FSN. Then 1 TA cell makes 100 boutons on FSNs $(10 \times 10=100)$. We then have that in total 1 TA cell makes 10,100 boutons in striatum (which is reasonable). TA makes a dense and specific innervation over a large area in striatum (Mallet et al., 2012); therefore, we did not put any spatial restriction on which striatal cells TA could make contact with. For TI to FSN, we likewise assumed that each FSN received input from $10 \mathrm{Tl}$ cells.

Both TA and TI neurons receive input from STN (Kita and Kitai, 1994), and TI gives rise to four times more collaterals than TA (Mallet et al., 2012). There are three TI neurons for each TA neuron in GPe $(75 \%$ TI versus $25 \%$ TA in GPe). So for each TI connection, we have $12(4 \times 3)$ as many TI connections onto an average GPe cell compared with a TA connection. It is reasonable to then assume that the majority of synapses onto specifically the TA cells come from $\mathrm{TI}$ neurons, since TI synapses are a magnitude more numerous than TA. However, it is still possible that TA neurons receive more connections from neighboring TA if there is some sort of connection preference rule at hand. Our base estimate in this study was that of 30 incoming GPe collaterals, 5 will be from TA and 25 from TI. Later in this article, we challenge this assumption to see the effect on network dynamics with different fan-in from $\mathrm{TI}$ and TA to TA.

\section{Synapse models}

To model static synapses, we used a standard conductance-based exponential decay model equation and the Tsodyks-Markram model (Tsodyks et al., 1998) to capture synaptic short-term plasticity (Hanson and Jaeger, 2002; Sims et al., 2008; Connelly et al., 2010; Gittis et al., 2010; Planert et al., 2010).

For static synapses, Eq. 3 is used when a presynaptic spike arrives. The conductance $g$ is updated with $g_{0}$ and then, in between the spikes, the conductance decays toward zero with time constant $\tau_{\text {syn }}$. The postsynaptic current is given by $I=g\left(E_{\text {rev }}-V\right)$, where $V$ is the membrane potential:

$$
\frac{d g}{d t}=-\frac{g}{\tau_{\text {syn }}}+g_{0} \delta\left(t-t_{\text {spike }}\right)
$$

The NMDA-dependent magnesium block was captured by multiplying the synaptic current with $B$ (Eq. 4; Humphries et al., 2009b):

$$
B(v)=\frac{1}{1+\frac{\left[\mathrm{Mg}^{2+}\right]_{2}}{3.57} \exp (-v 0.062)}
$$

To model a frequency-dependent synapse (Table 8), the Tsodyks model (Tsodyks et al., 1998) was used (Eqs. 5 and ${ }^{6}$ ) with the common FD formalism (Abbott et al., 1997; Dittman et al., 2000; Abbott and Regehr, 2004; Puccini et al., 2007). The FD formalism dictates that the synaptic strength is updated by the product of facilitating (F) and depressing (D) variables/factors. This description shows quantitatively good approximations of experimen- tally measured synapse dynamics (Tsodyks and Markram, 1997; Markram et al., 1998; Planert et al., 2010; Klaus et al., 2011; Lindahl et al., 2013). The model formalism assumes a finite pool of synaptic resources in active $(y)$, inactive ( $z$ ), and recovered $(x)$ states. At rest, $y$ and $z$ are 0 and $x$ is 1 . Depression occurs because some of the resources remain for a while in the inactive state before entering the recovered state, with a rate determined by the recovery time constant $\tau_{\text {rec }}$. The facilitation is modeled by $u$, which is a variable that is stepwise increased at each spike with the product of the utilization factor $U$ and $1-U$ ( $U$ is between 0 and 1 ) and decays exponentially toward 0 with time constant $\tau_{f a c}$ in between spikes (Eq. 5). The resources in the active state $y$ are increased with the product of the variables $x$ and $u$ (capturing depression and facilitation, respectively) and are then quickly inactivated by decaying toward zero with time constant $\tau_{\text {syn }}$ (Eq. 6). The postsynaptic conductance is proportional to the fraction of resources in the active state and is given by $g=$ $g_{0} y$, with the resulting postsynaptic current $I_{\text {syn }}=g$ $\left(E_{\text {rev }}-V\right)$ :

$$
\begin{gathered}
\frac{d u}{d t}=-\frac{u}{\tau_{\text {fac }}}+U(1-u) \delta\left(t-t_{\text {spike }}\right), \\
\frac{d x}{d t}=\frac{z}{\tau_{\text {rec }}}-u x \delta\left(t-t_{\text {spike }}\right) \\
\frac{d y}{d t}=-\frac{y}{\tau_{\text {syn }}}+u x \delta\left(t-t_{\text {spike }}\right) \\
\frac{d z}{d t}=\frac{y}{\tau_{\text {syn }}}-\frac{z}{\tau_{\text {rec }}} .
\end{gathered}
$$

Several of the synaptic parameters (Tables 7 and 8) were obtained from Lindahl et al. (2013). Below we explain how we derived CTX-MSN and the parameters from and to GPe TA and GPe TI. From Ellender et al. (2011), we estimate that the mean conductance evoked when stimulating cortical MSN fibers to $1.1 \mathrm{nS}$, since the peak of excitatory postsynaptic potential (EPSC) was $\sim 90 \mathrm{pA}$ at holding potential $-80 \mathrm{mV}$, with AMPA reversal potential at $0 \mathrm{mV}(90 \mathrm{pA} \div 80 \mathrm{mV}=1.1 \mathrm{nS})$. Assuming that the unitary strength of an AMPA synapse is below measured mean, we set it to $0.5 \mathrm{nS}$. Moyer et al. (2007) have estimated that the ratio of conductance sizes between AMPA and NMDA is $2: 1$. The conductance size is estimated here by the time constant multiplied with the peak conductance of a synapse; thus for the MSN AMPA synapse with a synaptic decay constant of $12 \mathrm{~ms}$ (Ellender et al., 2011), the conductance size equals $6(0.5 \times 12)$, and for the MSN D2 NMDA synapse with a synaptic time constant of $160 \mathrm{~ms}$ (Moyer et al., 2007; Humphries et al., 2009b), the maximal conductance has to be 0.019 , since the conductance size for NMDA should equal $3(0.019 \times 160)$, to give an AMPA and NMDA conductance size ratio of 2:1. Furthermore, Humphries et al. (2009a) estimated the parameter used for MSN D1 NMDA as $\sim 6$ times that for MSN D2 NMDA under normal dopamine conditions to resemble the behavior seen in more detailed MSN models. Thus we set 
Table 7. Basic synaptic model parameters

\begin{tabular}{|c|c|c|}
\hline Name & Value & Source \\
\hline$\tau_{\text {ampa }}^{C T X-F S N}$ & $12 \mathrm{~ms}$ & n.d., set as for CTX-MSN \\
\hline$g_{a m p a}^{C T X-F S N}$ & $0.5 \mathrm{nS}$ & n.d., set as for CTX-MSN \\
\hline$t_{\text {delay }}^{C T X-F S N}$ & $2.5 \mathrm{~ms}$ & Jaeger and Kita, 2011 \\
\hline$E_{\text {rev }}^{C T X-F S N}$ & $0 \mathrm{mV}$ & n.d., set as for CTX-MSN \\
\hline$\tau_{\text {gaba }}^{F S N-F S N}$ & $6 \mathrm{~ms}$ & Gittis et al., 2010 \\
\hline$g_{g a b a}^{F S N-F S N}$ & $1 \mathrm{nS}$ & Several times weaker than FSN-MSN (Gittis et al., 2010) \\
\hline$t_{\text {delay }}^{F S N-F S N}$ & $1.7 \mathrm{~ms}$ & n.d., same as FSN-MSN \\
\hline$E_{\text {rev }}^{F S N-F S N}$ & $-74 \mathrm{mV}$ & n.d., same as MSN-MSN \\
\hline$\tau_{g a b a}^{G P e_{T I}-F S N}$ & $17 \mathrm{~ms}$ & Saunders et al., 2016 \\
\hline$g_{\text {gaba }}^{G P e_{T I}-F S N}$ & $2 \mathrm{nS}$ & n.d., estimated \\
\hline$\tau_{g a b a}^{G P e_{T A}-F S N}$ & $66 \mathrm{~ms}$ & Glajch et al., 2016 \\
\hline$g_{g a b a}^{G P e_{T A}}-F S N$ & $0.51 \mathrm{nS}$ & n.d., estimated \\
\hline$t_{\text {delay }}^{G P e-F S N}$ & $7.0 \mathrm{~ms}$ & n.d., same as MSN-GPe Park et al., 1982 \\
\hline$E_{r e v}^{G P e-F S N}$ & $-74 \mathrm{mV}$ & n.d., same as MSN-MSN \\
\hline$\tau_{\text {ampa }}^{E X T-G P e}$ & $5 \mathrm{~ms}$ & n.d. \\
\hline$g_{g a b a}^{E X T-G P e}$ & $0.5 \mathrm{nS}$ & n.d. \\
\hline$t_{\text {delay }}^{E X T-G P e}$ & $5 \mathrm{~ms}$ & n.d. \\
\hline$E_{\text {rev }}^{E X T-G P e}$ & $0 \mathrm{mV}$ & n.d. \\
\hline$\tau_{g a b a}^{G P e-G P e}$ & $5 \mathrm{~ms}$ & Shen et al., 2008 \\
\hline$g_{g a b a}^{G P e-G P e_{T I}}$ & $1.3 \mathrm{nS}$ & Lindahl et al., 2013 \\
\hline$g_{g a b a}^{G P e-G P e_{T A}}$ & $0.33 \mathrm{nS}$ & $25 \%$ of $\mathrm{GPe}-\mathrm{GPe} \mathrm{TI}$ \\
\hline$t_{\text {delay }}^{G P e-G P e}$ & $1 \mathrm{~ms}$ & n.d. \\
\hline$E_{\text {rev }}^{G P e-G P e}$ & $-65 \mathrm{mV}$ & n.d., assumed as for MSN D2-GPe \\
\hline$\tau_{g a b a}^{M S N_{D 2}-G P e}$ & $6 \mathrm{~ms}$ & Shen et al., 2008 \\
\hline$g_{g a b a}^{M S N_{D 2}-G P e}$ & $2 \mathrm{nS}$ & Constrained by Shen et al., 2008 \\
\hline$t_{\text {delay }}^{M M_{D}-G P e}$ & $7 \mathrm{~ms}$ & Park et al., 1982 \\
\hline$E_{r e v}^{M S N_{D 2}-G P e}$ & $-65 \mathrm{mV}$ & Rav-Acha et al., 2005 \\
\hline$\tau_{\text {ampa }}^{S T N-G e_{T I}}$ & $12 \mathrm{~ms}$ & Hanson and Jaeger, 2002 \\
\hline$g_{\text {ampa }}^{S T N-G P e_{T I}}$ & $0.35 \mathrm{nS}$ & Lindahl et al., 2013 \\
\hline$g_{\text {ampa }}^{S T N-G P e_{T A}}$ & $0.11 \mathrm{nS}$ & $30 \%$ of STN-GPe TI \\
\hline$t_{\text {delay }}^{S T N-G P e_{T l}}$ & $2 \mathrm{~ms}$ & Jaeger and Kita, 2011 \\
\hline$E_{r e v}^{S T N-G P e_{T l}}$ & $0 \mathrm{mV}$ & n.d. \\
\hline$\tau_{\text {ampa }}^{C T X-M S N}$ & $12 \mathrm{~ms}$ & Ellender et al., 2011 \\
\hline$g_{\text {ampa }}^{C T X-M S N_{D 1}}$ & $0.5 \mathrm{nS}$ & Humphries et al., 2009a,b; Ellender et al., 2011 \\
\hline$g_{\text {ampa }}^{C T X-M S N_{D 2}}$ & $0.5 \mathrm{nS}$ & Humphries et al., 2009a,b; Ellender et al., 2011 \\
\hline$\tau_{n m d a}^{C T X-M S N}$ & $160 \mathrm{~ms}$ & Humphries et al., 2009a,b; Ellender et al., 2011 \\
\hline$g_{\text {nmda }}^{C T X-M S N_{D 1}}$ & $0.11 \mathrm{nS}$ & Humphries et al., 2009a,b; Ellender et al., 2011 \\
\hline$g_{n m d a}^{C T X-M S N_{D 2}}$ & $0.019 \mathrm{nS}$ & Humphries et al., 2009a,b; Ellender et al., 2011 \\
\hline$t_{\text {delay }}^{C T X-M S N}$ & $2.5 \mathrm{~ms}$ & Jaeger and Kita, 2011 \\
\hline$E_{r e v}^{C T X-M S N}$ & $0 \mathrm{mV}$ & Humphries et al., 2009a \\
\hline$\tau_{\text {gaba }}^{M S N-M S N}$ & $8 \mathrm{~ms}$ & Gittis et al., 2010 \\
\hline$g_{g a b a}^{M S N_{D 1}-M S N_{D 1}}$ & $0.15 \mathrm{nS}$ & Constrained by Koos et al., 2004; Taverna et al., 2004, 2008 \\
\hline$g_{g a b a}^{M S N_{D 1}-M S N_{D 2}}$ & $0.375 \mathrm{nS}$ & Constrained by Koos et al., 2004; Taverna et al., 2004, 2008 \\
\hline$g_{g a b a}^{M S N_{D 2}-M S N_{D 1}}$ & $0.45 \mathrm{nS}$ & Constrained by Koos et al., 2004; Taverna et al., 2004, 2008 \\
\hline$g_{g a b a}^{M S N_{D 2}-M S N_{D 2}}$ & $0.35 \mathrm{nS}$ & Constrained by Koos et al., 2004; Taverna et al., 2004, 2008 \\
\hline$t_{\text {delay }}^{\text {MSN-MSN }}$ & $1.7 \mathrm{~ms}$ & Taverna et al., 2004 \\
\hline$E_{r e v}^{M S N-M S N}$ & $-74 \mathrm{mV}$ & Koos et al., 2004 \\
\hline$\tau_{g a b a}^{F S N-M S N}$ & $11 \mathrm{~ms}$ & Koos et al., 2004 \\
\hline$g_{g a b a}^{F S N-M S N}$ & $6 \mathrm{nS}$ & Taverna et al., 2004 \\
\hline$t_{\text {delay }}^{F S N-M S N}$ & $1.7 \mathrm{~ms}$ & n.d., assumed as for MSN \\
\hline$E_{r e v}^{F S N-M S N}$ & $-74 \mathrm{mV}$ & $\begin{array}{r}\text { Koós and Tepper, } 1999 \\
\text { (Continued) }\end{array}$ \\
\hline
\end{tabular}


Table 7. Continued

\begin{tabular}{|c|c|c|}
\hline Name & Value & Source \\
\hline$\tau_{g a b a}^{G P e_{T A}-M S N_{D 1}}$ & $87 \mathrm{~ms}$ & Glajch et al., 2016 \\
\hline$\tau_{g a b a}^{G P e_{T A}}-M S N_{D 2}$ & $76 \mathrm{~ms}$ & Glajch et al., 2016 \\
\hline$g_{g a b a}^{G P e-M S N_{D 1}}$ & $0.04 \mathrm{nS}$ & n.d., estimated \\
\hline$g_{g a b a}^{G P e-M S N_{D 2}}$ & $0.08 \mathrm{nS}$ & Twice as TA-MSN D1 (Glajch et al., 2016) \\
\hline$t_{\text {delay }}^{G P e-M S N}$ & $7.0 \mathrm{~ms}$ & n.d., set as for MSN-GPe (Park et al., 1982) \\
\hline$E_{g a b a}^{G P e-M S N}$ & $-74 \mathrm{mV}$ & n.d., set as for MSN and FSN synapses \\
\hline$\tau_{\text {ampa }}^{\text {EXT-SNr }}$ & $5 \mathrm{~ms}$ & n.d. \\
\hline$g_{\text {ampa }}^{E X T-S N r}$ & $0.5 \mathrm{nS}$ & n.d. \\
\hline$t_{\text {delay }}^{E X T}-S N r$ & $5 \mathrm{~ms}$ & n.d. \\
\hline$E_{r e v}^{E X T-S N r}$ & $0 \mathrm{mV}$ & n.d. \\
\hline$\tau_{g a b a}^{M S N_{D 1}-S N r}$ & $5.2 \mathrm{~ms}$ & Connelly et al., 2010 \\
\hline$g_{g a b a}^{M S N_{D 1}-S N r}$ & $2 \mathrm{nS}$ & Constrained by Connelly et al., 2010 \\
\hline$t_{\text {delay }}^{M S N_{1}}-S N r$ & $7 \mathrm{~ms}$ & Connelly et al., 2010 \\
\hline$E_{r e v}^{M S N_{D 1}-S N r}$ & $-80 \mathrm{mV}$ & Connelly et al., 2010 \\
\hline$\tau_{g a b a}^{G P e-S N r}$ & $2.1 \mathrm{~ms}$ & Connelly et al., 2010 \\
\hline$g_{g a b a}^{G P e-S N r}$ & $76 \mathrm{nS}$ & Connelly et al., 2010 \\
\hline$t_{\text {delay }}^{G P e-S N r}$ & $3 \mathrm{~ms}$ & Nakanishi et al., 1991 \\
\hline$E_{r e v}^{G P e-S N r}$ & $-72 \mathrm{mV}$ & Connelly et al., 2010 \\
\hline$\tau_{\text {ampa }}^{S T N-S N r}$ & $12 \mathrm{~ms}$ & n.d., assume as for STN-GPe (Hanson and Jaeger, 2002) \\
\hline$g_{\text {ampa }}^{S T N-S N r}$ & $0.91 \mathrm{nS}$ & Lindahl et al., 2013 \\
\hline$t_{\text {delay }}^{S T N-S N r}$ & $4.5 \mathrm{~ms}$ & Shen and Johnson, 2006; Ammari et al., 2010 \\
\hline$E_{r e v}^{S T N-S N r}$ & $0 \mathrm{mV}$ & n.d. \\
\hline$\tau_{\text {ampa }}^{C T X-S T N}$ & $4 \mathrm{~ms}$ & Baufreton et al., 2005 \\
\hline$g_{\text {ampa }}^{\text {CTX-STN }}$ & $0.25 \mathrm{nS}$ & n.d. \\
\hline$\tau_{\text {nmda }}^{C T X-S T N}$ & $160 \mathrm{~ms}$ & Same as for MSN \\
\hline$g_{n m d a}^{C T X-S T N}$ & $0.00625 \mathrm{nS}$ & Same AMPA-NMDA ratio as for MSN \\
\hline$t_{\text {delay }}^{\text {CTX-STN }}$ & $2.5 \mathrm{~ms}$ & Fujimoto and Kita, 1993; Jaeger and Kita, 2011 \\
\hline$E_{\text {rev }}^{C T X-S T N}$ & $0 \mathrm{mV}$ & n.d. \\
\hline$\tau_{g a b a}^{G P e-S T N}$ & $8 \mathrm{~ms}$ & Baufreton et al., 2005 \\
\hline$g_{g a b a}^{G P e_{T I}-S T N}$ & $0.08 \mathrm{nS}$ & Lindahl et al., 2013 \\
\hline$t_{\text {delay }}^{G P e-S T N}$ & $1 \mathrm{~ms}$ & Jaeger and Kita, 2011 \\
\hline$E_{r e v}^{G P e-S T N}$ & $-84 \mathrm{mV}$ & Baufreton et al., 2009 \\
\hline
\end{tabular}

the MSN D1 NMDA to $0.11(0.019 \times 6)$. These differences between how MSN D1 and D2 NMDA properties are represented in this phenomenological point neuron model should not be interpreted literally; rather, it captures the dopamine-dependent modulation of the direct and indirect pathways.

Experiments suggest that both TA and TI neurons connect to FSNs, whereas only TA neurons connect to MSNs (Mallet et al. 2012; Smith et al., 1998; Glajch et al. 2016; Saunders et al. 2016). Specifically, Glajch et al. (2016) showed, by light stimulation of GPe axons going to striatum, that the inhibitory postsynaptic currents (IPSCs) in FSNs were considerable larger than the IPSCs in MSN D1 and MSN D2 (600 vs. 60 and 134 pA), and Saunders et al. (2016) measured IPSCs in FSNs by light stimulation of axons from TI neurons and observed large IPSCs in FSNs (437 pA). Here we assume that TA neurons connect twice as strongly to MSN D2 than to MSN D1 and that TA and TI neurons connect strongly to FSNs (Table 7) in accordance with Glajch et al. (2016) and Saunders et al (2016). Additionally, we assume that the TA and TI synapses onto
FSN are depressing (Corbit et al., 2016; Glajch et al., 2016). Because we did not have an estimate of the parameters for the depression, we used the same parameters as for FSN to MSN (Table 8). We also point out that the synapse dynamics of TA and TI neurons in striatum seem to differ. Saunders et al. (2016) recorded significantly faster synapse dynamics for TI cells onto FSNs compared with those Glajch et al. (2016) measured for the synaptic response in FSN, MSN D1, and MSN D2 when stimulating $\mathrm{GPe}$ axons (17 vs. 66,87 , and $76 \mathrm{~ms}$ ). The slice experiments in Glajch et al. (2016) were conducted at room temperature $\left(\sim 21^{\circ} \mathrm{C}\right)$, whereas Saunders et al. (2016) conducted their experiments at higher temperature $\left(\sim 31^{\circ} \mathrm{C}\right)$. It is commonly assumed that increased temperature can speed up such processes. Thus, the recording temperature could account for some of these differences. Here we assume that the synapse dynamics between TA and $\mathrm{TI}$ cells indeed differ and that TI synapses in striatum have faster dynamics than synapses from TA cells.

In Lindahl et al. (2013), the conductance between GPeGPe, GPe-STN, and STN-GPe neurons were respectively 
Table 8. Parameters for facilitating and depressing Tsodyks synapse models

\begin{tabular}{lllll}
\hline Synapse & $U$ & $\tau_{\text {rec }}$ & $\tau_{\text {fac }}$ & Source \\
$F S N-M S N$ & 0.29 & $902 \mathrm{~ms}$ & $53 \mathrm{~ms}$ & Planert et al., 2010 \\
$F S N-F S N$ & 0.29 & $902 \mathrm{~ms}$ & $53 \mathrm{~ms}$ & Gittis et al., 2010; Planert et al., 2010 \\
$G P e-S N r$ & 0.196 & $969 \mathrm{~ms}$ & $0 \mathrm{~ms}$ & Lindahl et al., 2013 \\
$M S N_{D 1}-S N r$ & 0.0192 & $623 \mathrm{~ms}$ & $559 \mathrm{~ms}$ & Lindahl et al., 2013 \\
$M S N_{D 2}-S N r$ & 0.24 & $11 \mathrm{~ms}$ & $73 \mathrm{~ms}$ & Lindahl et al., 2013 \\
$S T N-S N r$ & 0.35 & $800 \mathrm{~ms}$ & $0 \mathrm{~ms}$ & Lindahl et al., 2013 \\
$G P e_{T A}-F S N$ & 0.29 & $902 \mathrm{~ms}$ & $53 \mathrm{~ms}$ & n.d., assumed same as $F S N-M S N$ \\
$G P e_{T I}-F S N$ & 0.29 & $902 \mathrm{~ms}$ & $53 \mathrm{~ms}$ & n.d., assumed same as $F S N-M S N$
\end{tabular}

estimated to be $1.3,0.35$, and $0.08 \mathrm{nS}$. In this study, the $\mathrm{GPe} \mathrm{TI}$ neurons are assumed to correspond to the GPe neurons in Lindahl et al. (2013) since they have similar projection patterns. Thus GPe-GPe TI, GPe TI-STN, and STN-GPe TI were set to these values. TA neurons do not receive any inhibitory input from MSN D2. To maintain realistic firing rates in TA, we lowered STN-TA to $30 \%$ of STN-TI. However, to properly explain changes in $\mathrm{TI}$ and TA rates for slow-wave and activation, we also had to lower the weights of GPe-GPe TA to $25 \%$ of GPe-GPe TI.

Numerous connections in BG are modified by dopamine (Cepeda et al., 1993; Shen and Johnson, 2000; Bracci et al., 2002; Hernández-Echeagaray et al., 2004; Hernández et al., 2006; Baufreton and Bevan, 2008; Taverna et al., 2008; Chuhma et al., 2011; Gittis et al., 2011; Miguelez et al., 2012). The dopamine parameters related to the synaptic connectivity are listed in Table 9. Below we describe how we adapted the model to this.

\section{Dopamine effects on synapses onto FSNs}

Dopamine has a weakening effect on GABA synapses (Bracci et al., 2002), by activation of D2 receptors. We modeled this by multiplying $I_{G A B A}$ for FSN-FSN and GPe-FSN with, respectively, $1+\beta_{l}^{F S N-F S N} \phi$ and $1+$

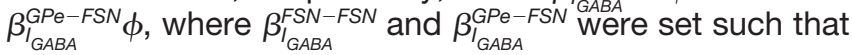
the IPSC amplitude at low dopamine $\left(\alpha_{\text {dop }}=0\right)$ were 2.7 (Humphries et al., 2009b) and 1.6 (Glajch et al., 2016) times the IPSC amplitude at high dopamine $\left(\alpha_{d o p}=1\right)$.

Table 9. Synaptic dopamine parameters

\begin{tabular}{|c|c|c|}
\hline Name & Value & Source \\
\hline$\beta_{I_{G A B A}^{F S N}}^{F S N N}$ & -1.27 & Humphries et al., $2009 a$ \\
\hline$\beta_{l_{G A B A}^{G P e}-F S N}$ & -0.53 & Glajch et al., 2016 \\
\hline$\beta_{l_{G A B A}^{G P B A}-G P e}^{G A}$ & -0.83 & Miguelez et al., 2012 \\
\hline$\beta_{I_{G A B A}^{G A B A}}^{M S N_{D 2}-G P e_{T I}}$ & -0.83 & Chuhma et al., 2011 \\
\hline$\beta_{l_{\text {AMPA }}^{S T N-G P e}}$ & -0.45 & Hernández et al., 2006 \\
\hline$\beta_{I_{N M D A}^{C T X}}^{C T S N_{D 1}}$ & 1.04 & Humphries et al., 2009b \\
\hline$\beta_{I_{A M P A}}^{C T X A}-M S N_{D 2}$ & -0.26 & Humphries et al., 2009b \\
\hline$\beta_{N_{\text {fan in }}}^{F S N-M S N_{D 2}}$ & -0.90 & Gittis et al., 2011 \\
\hline$\beta_{l_{G A B A}^{M S N-M S N}}^{M}$ & 0.88 & Taverna et al., 2008 \\
\hline$\beta_{N_{\text {tan in }}}^{M S N}-M S N$ & 0.88 & Taverna et al., 2008 \\
\hline$\beta_{G A B A}^{G P e_{T A}}-M S N_{D 1}$ & -1.22 & Glajch et al., 2016 \\
\hline$\beta_{I_{G A B A}^{G A B A}}^{G P e_{T A}-M S N_{D 1}}$ & -1.15 & Glajch et al., 2016 \\
\hline$\beta_{l_{G A B A}^{G A B A}}^{M S N_{D 1}-S N r}$ & 0.56 & Chuhma et al., 2011 \\
\hline$\beta_{l_{A M P A}^{C T X-S T N}}^{C T N}$ & -0.45 & Shen and Johnson, 2000 \\
\hline$\beta_{l_{G A B A}^{G P P A}}^{G A P T N}$ & -0.24 & Baufreton and Bevan, 2008 \\
\hline
\end{tabular}

\section{Dopamine effects on synapses onto GPe neurons}

Dopamine depletion-dependent weakening of GPeGPe synapses has been shown experimentally (Miguelez et al., 2012). This does not seem to involve dopamine receptor effects; instead, it is hypothesized that the change is due to maladaptive homeostasis. We modeled this by multiplying $I_{G A B A}$ for GPe-GPe with $1+$

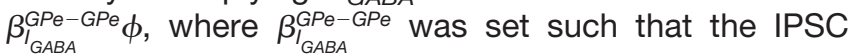
amplitude at low dopamine $\left(\alpha_{\text {dop }}=0\right)$ was two times the IPSC amplitude at high dopamine $\left(\alpha_{\text {dop }}=1\right)$ estimated from Miguelez et al. (2012).

It has been shown that dopamine leads to a decrease in synaptic efficacy of MSN-GPe synapses through D2 activation in rats (Ingham et al., 1997; Cooper and Stanford, 2001; Chuhma et al., 2011). We modeled this by multiplying $I_{G A B A}$ for MSN D2-GPe TI with $1+\beta_{I_{G A B}}^{M S N_{D 2}-G P e_{T 1}} \phi$, where $\beta_{l_{G A B A}}^{M S N_{D 2}-G P e_{T 1}}$ was set such that the IPSC amplitude at low dopamine $\left(\alpha_{\text {dop }}=0\right)$ was two times the IPSC amplitude at high dopamine $\left(\alpha_{d o p}=1\right)$ estimated from Chuhma et al. (2011).

Dopamine administration reduces STN-GPe EPSCs (Hernández et al., 2006). We modeled this by multiplying $I_{A M P A}$ for all STN-GPe synapses with $1+\beta_{I_{A M P A}^{S T N-G P e}} \phi$, where $\beta_{I_{A M P A}^{S T N-G P e}}$ was set such that the EPSC amplitude at low dopamine $\left(\alpha_{\text {dop }}=0\right)$ was 1.5 times the EPSC amplitude at high dopamine $\left(\alpha_{\text {dop }}=1\right)$ as estimated from Hernández et al., (2006).

\section{Dopamine effects on synapses onto MSNs}

Dopamine has a strengthening effect on CTX-MSN D1 NMDA currents, whereas it has a weakening effect on CTX-MSN D2 AMPA currents (Cepeda et al., 1993; Levine et al., 1996; Hernández-Echeagaray et al., 2004). We modeled this by multiplying $I_{N M D A}$ for CTX-MSN D1 by $1+\beta_{I_{N M D A}^{C T X}-M S N_{D 1}} \phi$ and multiplying $I_{A M P A}$ for CTX-MSN D2 by $1+\beta_{I_{A M P A}^{C T X}-M S N_{D 2}}^{C} \cdot \beta_{I_{N M D A}^{C T X}-M S N_{D 1}}^{C}$ was set such that the EPSC amplitude at low dopamine $\left(\alpha_{\text {dop }}=0\right)$ was 0.14 times the EPSC amplitude at high dopamine $\left(\alpha_{\text {dop }}=1\right)$, and $\beta_{I_{A M P A}^{C T X}-M S N_{D 2}}^{C}$ was set such that the EPSC amplitude at low dopamine $\left(\alpha_{\text {dop }}=0\right)$ was 1.27 times the EPSC amplitude at high dopamine $\left(\alpha_{\text {dop }}=1\right)$; both parameter values were obtained from Humphries et al. (2009b).

Dopamine depletion leads to an increase in connections between FSN-MSN D2 but not FSN-MSN D1 (Gittis et al., 2011). We modeled this by multiplying $N_{F S N-M S N_{D 2}}$ by $1+\beta_{N_{F S N-M S N_{D 2}}} \phi$ such that the number of connections at low dopamine $\left(\alpha_{\text {dop }}=0\right)$ was two times the number of 
connection at basal dopamine $\left(\alpha_{\text {dop }}=0.8\right)$ as estimated from Gittis et al. (2011).

Dopamine depletion leads to a dramatic decrease in the connectivity between MSNs (Taverna et al., 2008). We modeled this by multiplying $I_{\text {GABA }}$ for MSN-MSN by $1+$ $\beta_{I}^{M S N-M S N} \phi$ and multiplying $N_{M S N-M S N}$ for MSN-MSN by $1 \stackrel{\text { GABA }}{+} \beta_{N_{M S N-M S N}} \phi . \beta_{l_{\text {GABA }}^{M S M N}}$ was set such the IPSC at low dopamine $\left(\alpha_{\text {dop }}=0\right)$ was 0.25 times the size of the IPSC at high dopamine $\left(\alpha_{d o p}=1\right)$, and $\beta_{N_{M S N-M S N}}$ was set such that the number of MSN collaterals at low dopamine $\left(\alpha_{\text {dop }}=0\right)$ were 0.25 times the number MSN collaterals at high dopamine $\left(\alpha_{d o p}=1\right)$; both parameters were estimated from Taverna et al. (2008).

Dopamine has an weakening effect on TA-MSN synapses (Glajch et al., 2016). We modeled this by multiplying $I_{\text {GABA }}$ for TA-MSN D1 and TI-MSN D2 with, respectively, $1+\beta_{l_{G A B A}}^{G P_{T A}-M S N_{D 1} \phi}$ and $1+\beta_{l_{G A B A}}^{G P_{T A}-M S N_{D 2} \phi}$, where

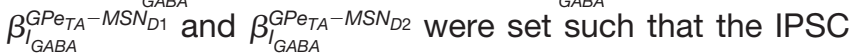
amplitude at low dopamine $\left(\alpha_{d o p}=0\right)$ was 2.6 and 2.5 times (Glajch et al., 2016) the IPSC amplitude at high dopamine $\left(\alpha_{\text {dop }}=1\right)$.

\section{Dopamine effects on synapses onto $\mathrm{SNr}$ neurons}

Dopamine D1 receptor activation facilitates MSN-SNr synapses (Chuhma et al., 2011). We modeled this by multiplying $I_{G A B A}$ with $1-\beta_{I_{G A B A}}^{M S N_{D 1}-S N r} \alpha_{d o p}$, where $\beta_{I_{G A B A}}^{M S N_{D 1}-S N r}$ was set such that the IPSC amplitude at low dopamine $\left(\alpha_{\text {dop }}=0\right)$ was 0.5 times the amplitude at high dopamine $\left(\alpha_{\text {dop }}=1\right)$ estimated from Chuhma et al. (2011).

Dopamine receptor D1 activation has a facilitating effect, whereas dopamine receptor D2 activation has a depressing effect on STN-SNr EPSC (Ibañez-Sandoval et al., 2006). Thus it is not clear whether dopamine enhances or weakens STN-SNr synapses. Here we assumed that dopamine activation did not change the amplitude of STN-SNr EPSC.

\section{Dopamine effects on synapses onto STN neurons}

Weakening of CTX-STN synapses by dopamine (Shen and Johnson, 2000) was modeled by multiplying $I_{A M P A}$ and $I_{\text {NMDA }}$ with $1-\beta_{I_{A M P A}^{C T X}-S T N} \phi$, where $\beta_{I_{A M P A}^{C T X}-S T N}$ was set such that EPSC amplitude at low dopamine $\left(\alpha_{\text {dop }}=0\right)$ was 2.5 times the amplitude at high dopamine $\left(\alpha_{d o p}=1\right)$ estimated from Kreiss et al. (1997) and Magill et al. (2001). With this value of $\beta_{l}^{\text {CTX-STN }}$, the firing rate of STN neurons in the network increased $\sim 100 \%$ when removing dopamine, which is in agreement with experiments (Magill et al., 2001; Mallet et al., 2008).

The results by Baufreton and Bevan (2008) suggest that dopamine causes a small but significant decrease in GPe-STN synaptic efficacy at $10-$ to $50-\mathrm{Hz}$ firing rate. This was modeled by multiplying the IPSC by 1 $\beta_{I_{G A B A}^{G P e}-S T N} \phi . \beta_{l_{G A B A}^{G P e}-S T N}$ was set such that the synaptic conductance without dopamine $\left(\alpha_{\text {dop }}=0\right)$ was 1.25 times the synaptic conductance at maximal dopamine level $\left(\alpha_{\text {dop }}=1\right)$ estimated from Baufreton and Bevan (2008).

\section{Data analysis}

Spike trains were sampled at $256 \mathrm{~Hz}$ as in Mallet et al. (2008) when computing coherence. The Hanning window was set to 128 and $2048 \mathrm{~ms}$ such that we got frequency resolution for cortical beta and slow-wave activity at 1 and $0.125 \mathrm{~Hz}$ as in Mallet et al. (2008). For significance levels for coherence, we used a method from Halliday et al. (1995).

To calculate the phase relation between two neurons, we first smoothed the raw spike trains with a bandpass filter at $0.5-1.5 \mathrm{~Hz}$ and $15-25 \mathrm{~Hz}$, respectively, for slowwave and beta activity. Then we applied the Hilbert transform on the smoothed data to obtain the instantaneous phases, and finally got the phase relation between two neurons by subtracting their instantaneous phases from each other. The result was plotted in a histogram with 100 bins between $-\pi$ and $\pi$.

Firing rate is calculated from time bins equaling 1000/ 256 ( $\sim 4 \mathrm{~ms}$; assuming the same sampling frequency as in Mallet et al, 2008).

To quantify synchrony in BG neurons, we used the Fano factor as a measure (Kumar et al., 2008, 2011). The Fano factor (Eq. 7) of a population $F F(p o p)$ is defined as the variance of the total population firing rate $V(p o p)$ divided by mean of the population firing rate $E(p o p)$. The sampling frequency in Mallet et al., (2008) was $256 \mathrm{~Hz}$; to match this, we used a bin size of 1000/256 $\mathrm{ms}(\sim 4 \mathrm{~ms})$ to calculate population firing rates. Poisson processes have a Fano factor of 1. A Fano factor $>1$ then means that some neurons must fire in a more synchronized manner, i.e., there are more spike events from different neurons occurring close in time than a Poisson process would predict:

$$
F F(p o p)=V[p o p] / E[p o p] .
$$

To estimate the strength of beta oscillations in a population, we used the fact that oscillations introduce peaks in the power spectrum density (Kumar et al., 2011). Power spectrum, $S$, was calculated from the average over the spectra of individual neuron spike trains. The oscillation index used to capture this, Ol, was defined as relative power in a frequency band:

$$
\text { O/[pop] }=\frac{\int_{a}^{b} S(f)_{p o p} d f}{\int_{0}^{F s / 2} S(f)_{p o p} d f},
$$

where $a$ and $b$ were set to respectively 15 and $25 \mathrm{~Hz}$, a typical frequency band for beta oscillation. The sample frequency Fs was $256 \mathrm{~Hz}$ as in Mallet et al. (2008).

\section{Implementation}

The simulations were run using the NEST simulator (Gewaltig and Diesmann, 2007; RRID:SCR_002963). Simulations ran on a CRAY XC30 system. The simulation of a network with 80,000 neurons for $10 \mathrm{~s}$ took $30 \mathrm{~min}$ on 80 cores. The network was built using PyNest, which is a Python interface to the NEST simulator. The model is available for download at github (https://github.com/ mickelindahl/bgmodel). 

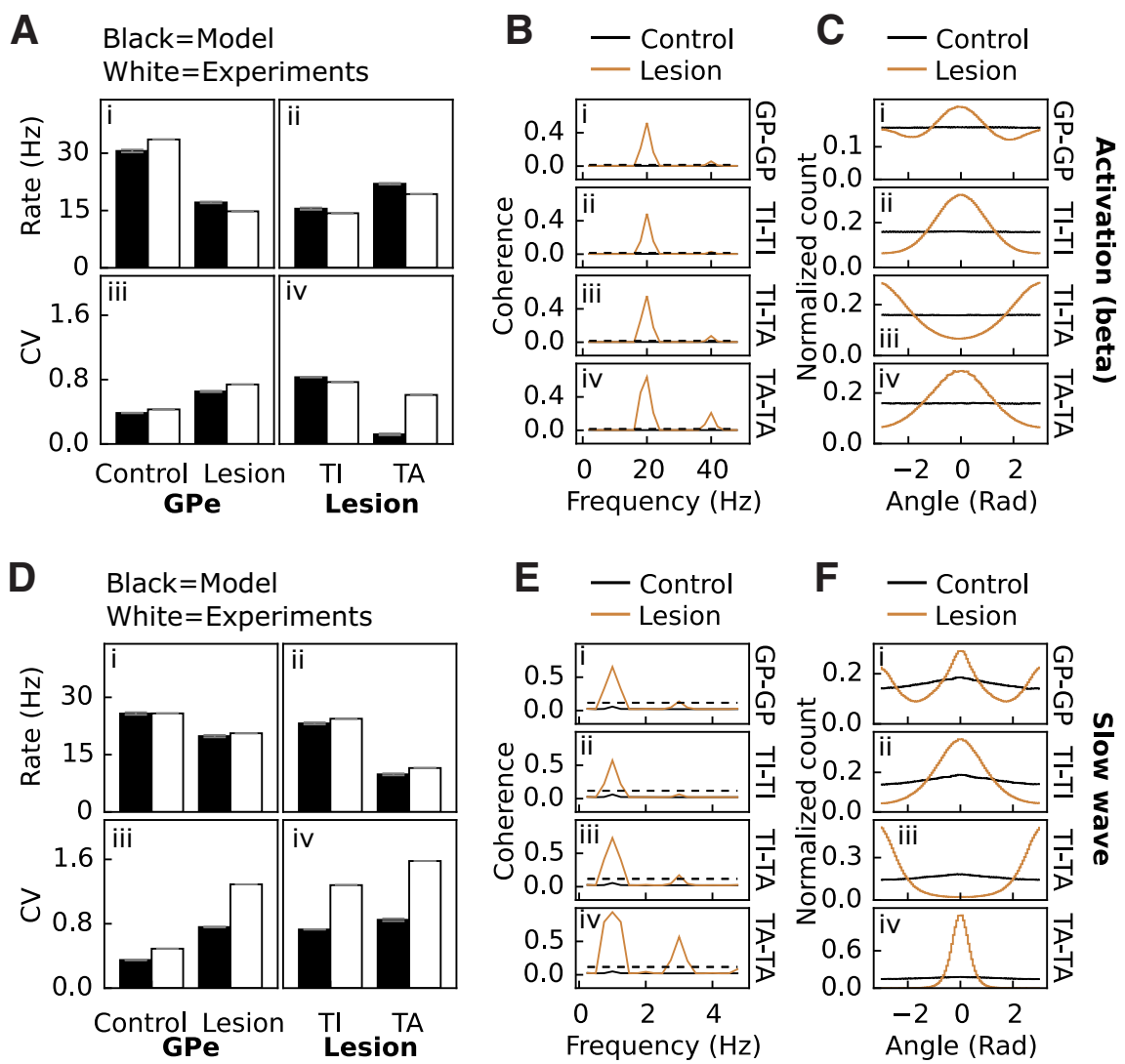

Figure 2. Model validation using GPe as a readout. $\boldsymbol{A}$, Firing rate and $\mathrm{CV}$ of GPe neurons with cortical beta activity used as input for the model, and comparisons with experiments (from Mallet et al, 2008). i, iii, Firing rate and CV of randomly selected GPe neurons in the control and lesioned (dopamine set to 0) network. ii, iv, Firing rate and CV for GPe TI and GPe TA neurons in the lesioned network. $\boldsymbol{B}$, Coherence of GPe neurons in the control and lesioned network when activated with cortical beta input. $\boldsymbol{i}$-iv, Coherence for random GPe versus GPe, specifically GPe TI versus GPe TI, GPe TI versus GPe TA, and GPe TA versus GPe TA. Black dotted line shows significance of $p=0.05$. $\boldsymbol{C}$, Phase relationship of GPe neurons in the control and lesioned network when activated with cortical beta input. $\boldsymbol{i}$-iv, Phase relationship for random GPe versus GPe, GPe TI versus GPe TI, GPe TI versus GPe TA, and GPe TA versus GPe TA. $\boldsymbol{D}, \boldsymbol{E}$, and $\boldsymbol{F}$, same as $\boldsymbol{A}, \boldsymbol{B}$, and $\boldsymbol{C}$, but for cortical slow-wave input.

\section{Results}

\section{Constraining and validating the systems-level BG model with statistics on GPe and STN firing rates, coefficients of variation, coherences, and phase relations}

A massive body of work in this study has been to review and compile model data from a large collection of experimental papers and integrate the information into a BG network model. First and foremost, we were concerned with constraining the model to experimental data on connectivity and nuclei sizes (Table 1), synaptic properties (Tables 7 and 8), neuron properties (Tables 2-6), and effects of dopamine depletion (Table 9 and Fig. 1C). Two models were used in this study, one with an intact dopamine system (control model) and one representing dopamine-depleted rats after oxidopamine (6-OHDA) treatment (lesioned model). In the current study, one goal was to validate the model against the statistics in Mallet et al. (2008) by comparing model data and experimental data regarding firing rates, coefficients of variation (CVs) of interspike intervals, coherence, and phase shift for and between STN and GPe TI and TA neurons.
In Mallet et al. (2008), the authors measured the spike statistics of GPe and STN neurons in rats under urethane anesthesia for two cortical states: during cortical activation, which was elicited by pinching the hindpaw, or during cortical slow-wave activity, which also resembles the activity observed during natural sleep. We found that GPe and STN firing rates for both cortical states could be reproduced by the model (Figs. $2 A i, D i$ and $3 A i, D i)$. The model could also capture the increase in CV of the GPe neurons between control and lesioned rats (Figs 2Aiii, Diii) for both cortical states as well as the decrease/increase in CV for STN neurons during activation (Fig. 3Aii). However, the weak increase in CV seen during slow-wave activity could not be reproduced (Fig. 3Dii).

The emergence of oscillations in BG and associated regions of the thalamus, caused by dopamine depletion, is a well-known phenomenon (Gatev et al., 2006). Mallet et al. (2008) showed significant coherence at 20 and $1 \mathrm{~Hz}$ for lesioned rats during both cortical states, both within and between GPe and STN neuron populations. The model could reproduce the coherence seen between randomly chosen GPe-GPe and between specifically chosen TI-TI, TI-TA, and TA-TA neurons (Figs. 2B, E) as well as 

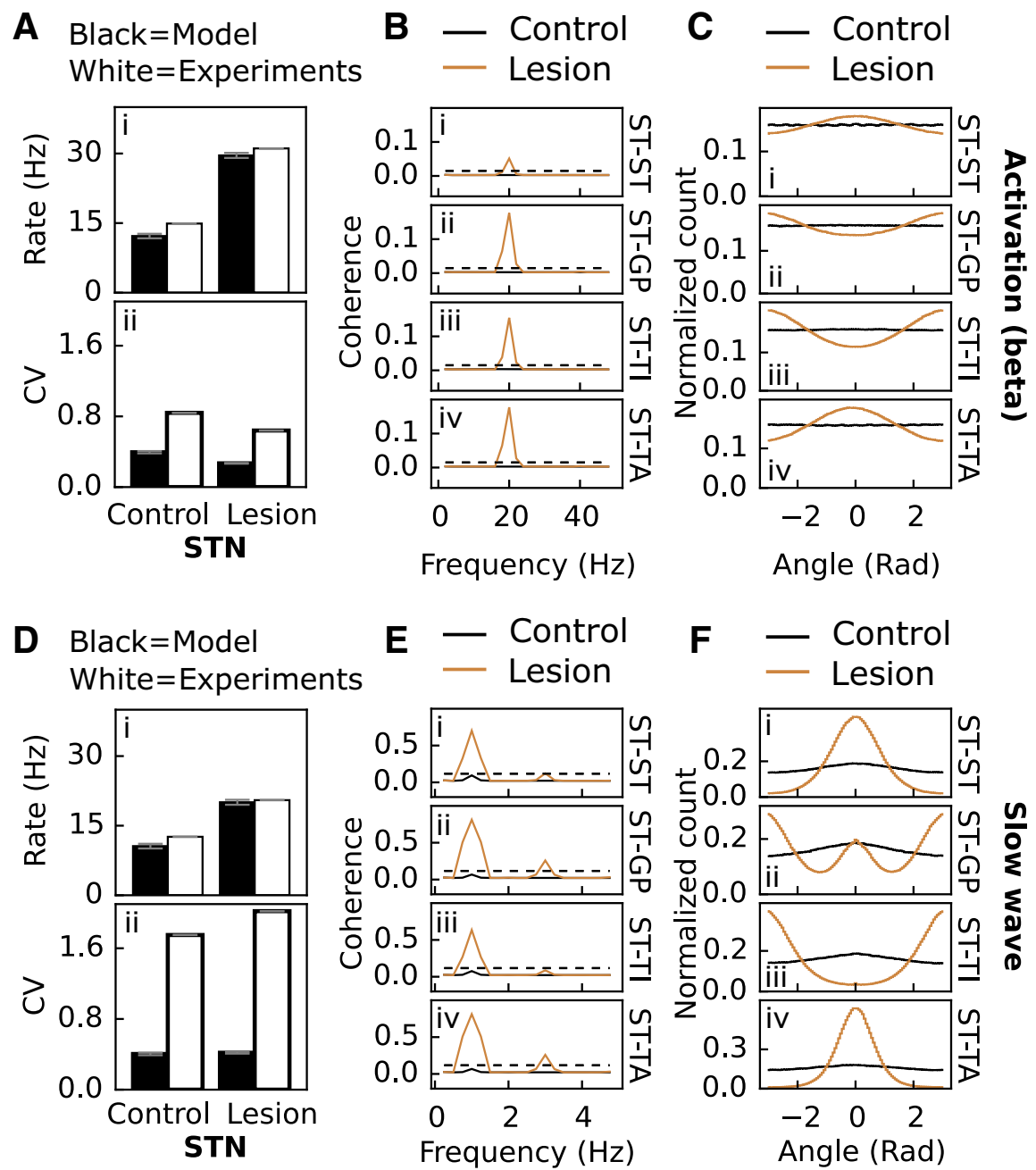

Figure 3. Model validation using STN as a readout. $\boldsymbol{A}$, Firing rate and CV of STN neurons with cortical beta input for model and experiment (Mallet, et al, 2008). i, ii, Firing rate and CV of STN neurons in control and lesioned (dopamine set to 0) network. B, Coherence of STN and GPe neurons in control and lesioned network with cortical beta input. i-iv, Coherence for STN versus STN, STN versus GPe, STN versus GPe TI, and STN versus GPe TA. Dotted black line shows significance of $p=0.05$. C, Phase relationship of STN and GPe neurons in control and lesioned network with cortical beta input. $\boldsymbol{i}$-iv, Phase relationship for STN versus STN, STN versus GPe, STN versus GPe TI, and STN versus GPe TA. $\boldsymbol{D}, \boldsymbol{E}$, and $\boldsymbol{F}$, same as $\boldsymbol{A}, \boldsymbol{B}$, and $\boldsymbol{C}$, for cortical slow-wave input.

between STN-STN, STN-GPe, STN-TI, and STN-TA neurons (Figs. 3B, E) for cortical activation and slow-wave activity in lesioned rats. After cortical activation in the control model, the coherence stays below the significance level (Figs. 2B and 3B, black lines), whereas in the lesioned model the coherence between the pairs goes well above significance level (Figs. $2 B$ and $3 B$, copper lines). Similar results were observed in simulations with cortical slow-wave activity (Figs. $2 E$ and $3 E$ )

The firing rate and CV relation of specifically the TA and TI neurons in lesioned rats found in Mallet et al. (2008) were also captured by the model. A puzzling observation that could prove difficult to reproduce by the model is that the firing rate of TI neurons are lower than TA neurons under cortical activation in dopamine-depleted rats (Fig. 2Aii), whereas under cortical slow-wave activity, the firing rate of TA neurons instead are higher than GPe TI neurons (Fig. 2Dii). It turns out that one solution to this puzzle had to do with the cortical and thalamic input to striatum, $\mathrm{GPe}$ and STN. We hypothesized that during cortical activation the input from cortex and thalamus is higher than during cortical slow-wave activity. Indeed, we found that hypothetically higher cortical and thalamic mean inputs during cortical activation than during cortical slow-wave activity could explain the TA and TI rate relation between the two cortical states (compare Fig. 2Aii, Dii). Also, the model reproduced qualitatively the observation in lesioned rats that the CVs of TI neurons were lower than CVs of TA neurons with cortical activation but then reversed with cortical slow-wave activity (compare Fig. 2Aiv, Div).

An interesting discovery in Mallet et al. (2008) was that $\mathrm{TI}$ and TA as well as STN and TI neurons in lesioned rats during both cortical states fired out of phase with each other, whereas STN and TA neurons fired in phase. We found that the model could reproduce this (TI-TA and STN-TI out of phase, Figs. 2Ciii, Fiii and 3Ciii, Fiii; STN-TA in phase, Fig. 3Civ, Fiv), and additionally that TI-TI, TATA, and STN-STN neurons in the model fire in phase 
A

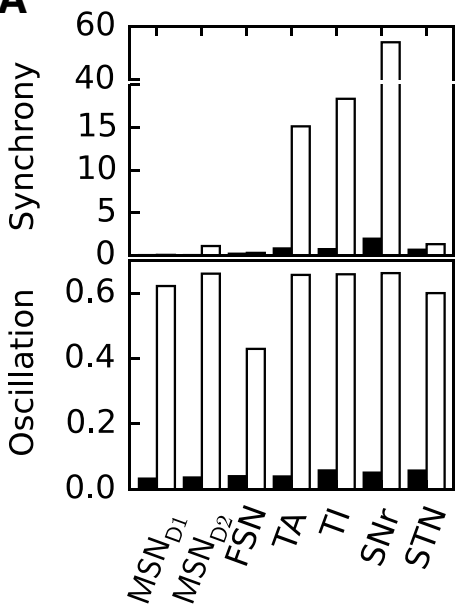

Black $=$ Control

White $=$ Lesion

\section{B}
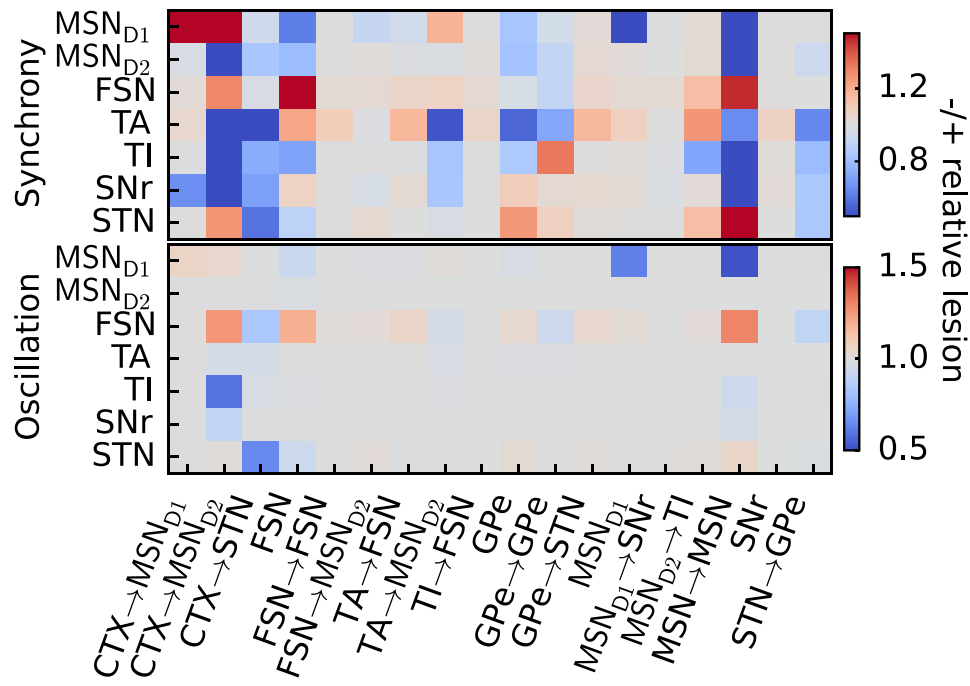

\section{C}
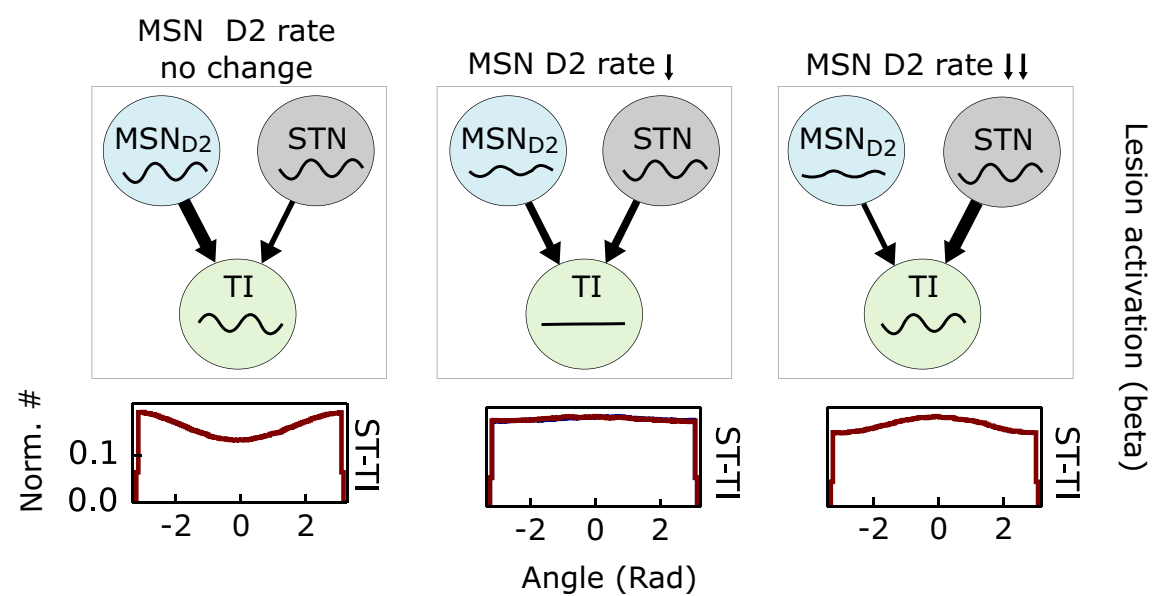

Figure 4. Local network dynamics and the role of dopamine-dependent perturbations. $\boldsymbol{A}$, Synchrony and oscillations in control and lesioned network components. Upper panel shows amount of synchrony in MSN D1, MSN D2, FSN, GPe TA, GPe TI, SNr, and STN during control (black bars) and after dopamine depletion (white bars). Lower panel shows the same but for oscillations instead of synchrony. B, Upper panel shows the relative change in synchrony in MSN D1, MSN D2, FSN, GPe TA, GPe TI, SNr, and STN ( $y$ axis) compared with the lesioned network when restoring the parameter on the $x$ axis to the value it had in the control network (no dopamine depletion). Lower panel shows the same but for oscillations instead of synchrony. $\boldsymbol{C}$, Phase relationship of STN and GPe TI when decreasing MSN D2 firing rate in the lesioned model. Cartoons illustrating effect of no change, medium decrease, and large decrease of MSN D2 firing rate. Signals represent instantaneous population firing rate in each nuclei.

during both cortical states (Fig. 2Cii, Civ, Fii, Fiv and $3 \mathrm{Ci}$, Fi) as seen in Mallet et al. (2008). The model could also reproduce the single peak-valley coherence for $\mathrm{GPe}-$ GPe/STN-GPe neurons under cortical activation (Fig. $2 \mathrm{Ci}$ and $3 \mathrm{Cii}$ ) and the double-valley coherence for the same pairs under cortical slow-wave activity (Fig. 2Fi and 3Fii). Thus the phase relationships for the GPe-STN network from Mallet et al. (2008) could be captured by the model.

\section{Mechanisms enhancing and quenching synchrony and oscillations in the dopamine-depleted state}

The model exhibits appropriate enhancement of oscillations and synchrony in the dopamine-depleted state. Fig. $4 A$ shows synchrony and oscillation index for all $B G$ nuclei, with cortical activation as input for the control and lesioned (dopamine-depleted) models. It can be seen by comparing the black and white bars that there are significant increases in most of the indexes for different neuron populations.

It is clear from experiments that dopamine depletion has a significant effect on multiple network components in $B G$, e.g., by perturbing the synaptic signaling, connectivity degree, or neural excitability (Fig. 1C). However, the relative importance of each perturbation for driving the observed network changes after dopamine depletion (Mallet et al., 2008) is still not well understood. Thus to test how each individual perturbation affects the dynamics of the whole network, we ran several simulations. For each of the model parameters assumed to be perturbed after dopamine depletion, we restored the parameter values to 
the control, one at a time, and compared the simulation results with the model in which all parameters were set to the dopamine-depleted value.

We found that changes to neural excitability and synaptic coupling involving components of the GPe-STN network due to dopamine depletion have a diverse effect on synchrony and oscillations, with some neuronal populations showing increases and some decreases for a change in a single dopamine parameter. For example, restoring GPe excitability or lateral GPe connectivity both decrease synchrony in TA, but the TI effects are opposite (Fig. 4B). Restoring the dopamine depletion-induced increase in CTX to STN synapses has a dampening effect on synchrony in TA, TI, SNr, and STN and on oscillations in STN. Thus the reduction in excitability of GPe neurons or increase in coupling between CTX and STN seen in experiments after dopamine depletions has a significant effect on both synchrony and oscillations over multiple $B G$ nuclei.

Restoring the perturbation on MSN D2-TI connectivity leads in our model to a decrease in synchrony in GPe TI neurons (Fig. $4 B$ ) in line with the results of Kumar et al. (2011). However, there is also a weak increase in synchrony for some other neuronal populations (Fig. 4B). Effects on oscillations are negligible.

Simulations suggested that restoring the perturbation to CTX-MSN D2 connectivity has a strong effect on synchrony and/or oscillations in BG with a decrease seen in MSN D2, SNr, TA, and TI (Fig. 4B) and an increase seen in MSN D1, FSN, and STN. A qualitatively similar effect in synchrony can also be seen when restoring the perturbation to MSN collaterals, except in MSN D1, where the result is the opposite.

We found it remarkable that restoring CTX-MSN D2 or MSN collaterals had such a dramatic effect on synchrony and/or oscillations throughout the network. Restoring both these perturbations decreases the firing rate in MSN D2 neurons. We hypothesize that oscillatory input from MSN D2 and STN competes in TI neurons. To test this, we ran three simulations with the lesioned network while varying the MSN firing rate. In Fig. $4 C$, it can be seen how the phase relation between STN-TI depends on MSN D2 firing rate, where a reduced firing rate of MSN D2 shifts the STN and TI neurons from out of phase, via cancelling each other, to in phase. In summary, the model results suggest that oscillatory cortical input conveyed via MSN D2 and STN competes in TI and that the effect can be balanced in TI. As the synchrony in TI goes down, TA and SNr also show less synchrony.

To summarize, we see that dopamine depletion-induced perturbations in general result in a dampening effect on synchrony and oscillations in some BG nuclei and a facilitating effect in others.

\section{Inhibitory control of MSNs and resulting effects for the striatal gating of cortical inputs}

Inhibition from FSNs and GPe TA is important for controlling MSN firing rate at low cortical activity, whereas collaterals from neighboring MSNs may become important at higher cortical input (when MSNs spike more). The
MSNs receive inhibitory input from three main sources, collaterals from neighboring MSNs, from FSNs, and GPe TA neurons. Earlier work has proposed that the weak, sparsely connected, and numerous MSN collaterals in the network set the overall excitability level (Wickens et al., 2007) and that strong feed-forward inhibition by FSNs acts as fast inhibition determining the moment-tomoment firing pattern of MSNs (Tepper et al., 2004). Thus two main players, collateral and feed-forward inhibition, have been considered important contributors to inhibition in striatum. But recently, a third player appeared, namely striatal inhibition from GPe (Mallet et al., 2012). Research showed that one GPe cell can form $\sim 10,000$ synapses in striatum and that GPe synapses constitute a significant amount of the total number of inhibitory synapses in striatum. Here, we wonder what the role of inhibition from $\mathrm{GPe}$ is, and how it compares to collateral and feedforward inhibition. To test this, we set up a simulation in which we increased the input from cortex to MSN, FSN, and STN in a stepwise fashion and measured the firing rate in a reduced striatal network with $\sim 3000 \mathrm{MSNs}$ for five different scenarios when it comes to connectivity. The rest of the network neural populations (FSN, STN, GPe, and $\mathrm{SNr}$ ) were also scaled down by a similar factor. We used $\sim 3000 \mathrm{MSNs}$, since that is the smallest striatal network in which MSN-MSN connection probabilities can be maintained. The result of Fig. $5 \mathrm{~A}$ was also confirmed in the full network model of 80,000 neurons. We ran simulations with no inhibition onto MSNs, inhibition from MSNs only, from FSNs only, from GPe TA only, and finally with full inhibitory connectivity (Fig. 5A). It can be seen that FSN and GPe are more important in controlling the firing rate of MSNs during low cortical input, whereas MSNs are more important at higher cortical input (Fig. 5A). Thus the role of GPe seems to be more in line with FSN, which for low cortical input may control MSN firing, whereas collaterals control MSN firing rate relatively more at higher cortical input, thus acting as a regulator of MSNs overall excitability. Note that in the dopamine-depleted network, the relative importance of MSN-MSN inhibition will be changed, as MSN-MSN synapses are weakened while the inhibition from both FSN and TA rather increases.

\section{Could the intrastriatal lateral inhibition be important for the control of action selection?}

As seen from the description above, activated MSNs that are not inhibited by collaterals from other active MSNs might have significantly higher firing rates. It has been proposed that the collateral network in striatum could work as a winner-take-all network (Wickens, 1997). For such a network to succeed, one has to assume strong connections between MSNs, but physiological evidence suggest that the connections are weak (Jaeger et al., 1994; Tunstall et al., 2002; Taverna et al., 2004; Tepper et al., 2004). To test whether weak connections between MSNs (0.15-0.45 nS) still can be important for controlling the local contrast between activated MSNs, a factor that could be important for action selection, we simulated two scenarios. We embedded in our reduced striatal network ( $\sim 3000$ neurons) a number of nonconnected clusters of 
A

$$
\begin{array}{ll}
\text { - All } & \text { Only } \mathrm{FSN} \rightarrow \mathrm{MSN}^{\text {static }} \\
\text { - Only MSN } \rightarrow \mathrm{MSN} \text { - Only } \mathrm{GPe}_{\mathrm{TA}} \rightarrow \mathrm{MSN}^{-} & \text {- No inhibition }
\end{array}
$$
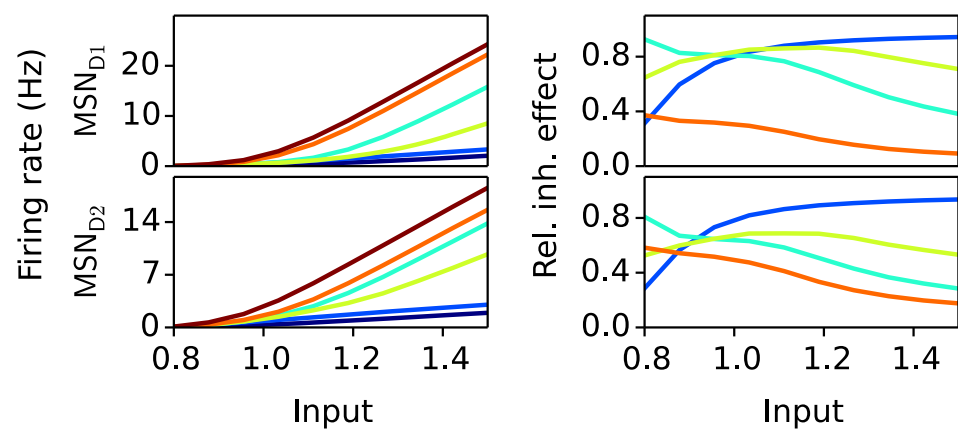

B
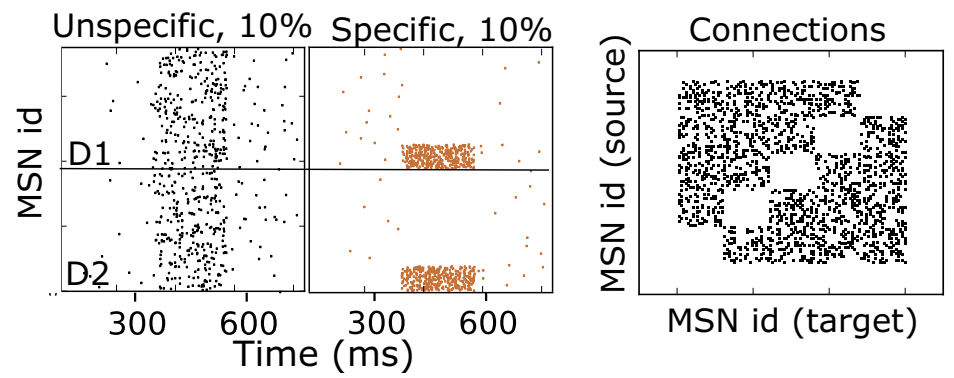

C
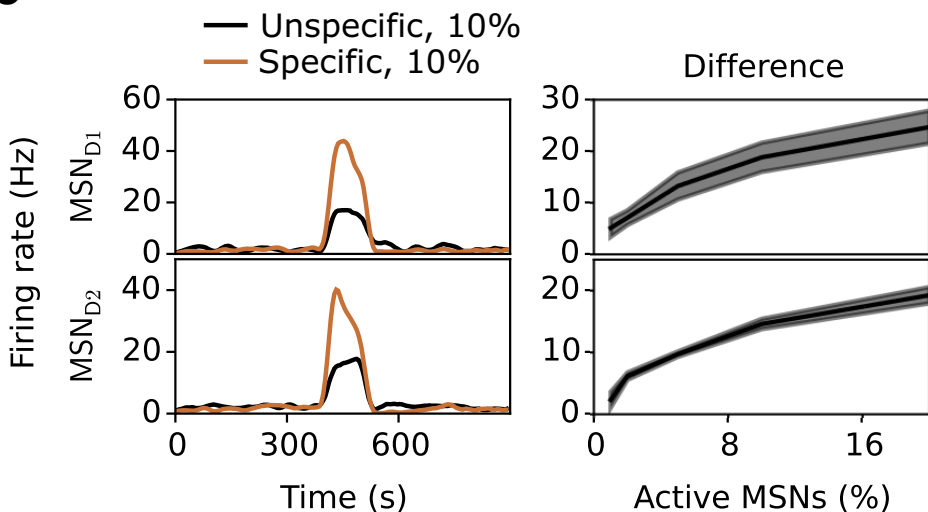

Figure 5. Control of firing rates of MSN neurons due to striatal inhibition. $\boldsymbol{A}$, Illustration how firing of MSN D1 and MSN D2 neurons are affected by inhibition from FSN, GPe TA, and MSN collaterals. Left top panel shows the firing rate of MSN D1 neurons with cortical input successively changed by a factor of 0.8 to 1.5 relative control, when keeping all inhibition (dark blue), only MSN-MSN inhibition (light blue), only FSN-MSN inhibition (cyan), only FSN-MSN static synapse inhibition (light green), only GPe TA-MSN inhibition (orange), and without any inhibition (dark red). Right top panel shows the relative contribution of the different sources of inhibition with successively increased cortical input and for MSN-MSN inhibition (light blue), FSN-MSN inhibition (cyan), FSN-MSN static synapse inhibition (light green), and GPe TA-MSN (orange) inhibition. Left and right bottom panels show the same as left and right top panels, respectively, except for MSN D2. B, Illustration of the role of lateral inhibition when a subpopulation, 10\%, of MSNs are activated. MSNs D1 and D2 are enumerated and given an index (id) between 1-1500. Left top panel shows the result when randomly selected MSN D1 neurons are activated; middle top panel shows the result when only nonconnected MSN D1 neurons are activated. Left and middle bottom panels show the same as left and middle top panels, except for MSN D2. Right panel shows the connection diagram for MSNs, in which a dot indicates a connection between MSNs. It illustrates how groups of MSNs, here those with low id, are not connected. $\boldsymbol{C}$, Left top panel shows the firing rate of the 10\% of MSN D1 activated with a 100-ms-long input burst for the case when randomly connected MSNs (blue) and specifically nonconnected MSNs (red) are targeted. Right top panel shows the mean difference between the firing rate of the two differently selected populations of MSN D1 neurons when varying the activated MSN population size between $2 \%$ and $20 \%$. Left and right bottom panels show the same thing as left and right top panels, except for MSN D2. 
MSN neurons (Fig. $5 B$ ). In the first scenario, we randomly activated neurons from the whole pool of MSNs neurons, and in the second scenario, we specifically activated only nonconnected MSNs. It is clear that nonconnected MSNs spike higher than connected (Fig. 5C, first panel) and that this holds for over a range of different percentage of activated MSNs (Fig. 5C, second panel). Weak collateral MSN connectivity may thus be able to control the firing rate of neighboring MSNs and work as a mechanism increasing the contrast during action selection scenarios. This effect is expected to be significantly larger in a control network compared with a dopamine-depleted network.

\section{Further support for the action selection hypothesis}

Kravitz et al. (2010) showed that MSN D1 activation promotes actions and MSN D2 activation inhibits actions. Cui et al. (2013) showed that both D1 and D2 MSNs are active in parallel during action selection experimental paradigms. These two studies suggest that populations of MSN D1 and MSN D2 that are activated by the same cortical activity state and are involved in the control of a resulting specific action do not project onto the same output neurons in SNr, since if they did, they would counteract each other based on the results of Kravitz et al. (2010). Therefore, we assume that the direct and indirect pathways converge on different SNr populations following co-activation by a specific cortical state that has been associated after learning with a certain action. To test whether the model supports the action selection hypothesis, we inserted two types of connectivity in the full model. The inserted connectivity, represented by activation of specific synapses between cortex and striatum, was assumed to have been learned from previous behavioral experiences. For models tackling this problem, see Potjans et al. (2011), Stewart et al. (2012), and Berthet et al. (2016). Strictly speaking, we tested with our model how suited BG are for transferring learned state action signals to motor-related output nuclei in thalamus or brainstem. However, this problem is not trivial. There is a high convergence of inhibitory MSN D1 connections on $\mathrm{SNr}(50: 1)$, potentially resulting in a scenario in which $\mathrm{SNr}$ neurons are easily overwhelmed by striatal inputs and become unable to differentiate between MSN inputs representing different actions associated with certain cortical states. Below we first see how the model can behave when only action signals are communicating though the direct pathway.

To test action selection capability, we set up two models. In the first model, "only D1," we included two equally sized action pools in the MSN D1 network connected to corresponding action mappings in SNr (Fig. 6A). In the second model, "D1 and D2," we also included action pools in the MSN D2 population that projected to corresponding pools in $\mathrm{GPe} \mathrm{TI}$, and finally the $\mathrm{GPe} \mathrm{Tl}$ projected to action mapping in $\mathrm{SNr}$ (Fig. 6A). It was assumed that the connectivity between cortex and striatum had been learned such that cortical state 1 (S1) activated a MSN D1 pool that in turn inhibited action $1 \mathrm{SNr}$ neurons and at the same time activated MSN D2 neurons that increased the activity of action $2 \mathrm{SNr}$ neurons (i.e., non-action $1 \mathrm{SNr}$ neurons) through the indirect pathway. See Berthet et al. (2016) for how such connectivity can be learned. The opposite connectivity was assumed for state 2 when activating MSN D1 and D2 neurons. An underlying assumption is that there exist MSN D1 and D2 populations that represent a Go and a NoGo signal for a specific action, and that the corticostriatal connectivity is learned such that a certain cortical state activates one striatal Go population as well as one or perhaps several NoGo populations suppressing opposing actions. This is in line with the results of Kravitz et al. (2010) and Cui et al. (2013), in which a specific cortical state activates D1 and D2 MSNs that project onto different SNr neurons. In both the first and second models, we also could vary the sizes of the action pools in D1 and D2 neurons, e.g., between 10\% and $100 \%$ of the corresponding neuron population type (see below). We thus formed what can be viewed as action channels through the BG (see Fig. 6A). Except for the assumed action channels, all other connectivities in the model were randomized.

Action selection should work over a range of different inputs and number of active presynaptic neurons. Humphries et al. (2006) showed how BG can perform action selection over a range of different activation rates of MSN neurons. Such properties are important for the dynamic range of the system. Imagine that we have two actions that are activated in a stepwise fashion at different firing rates, with one lower than the other (Fig. 6B). In the beginning, a good contrast can be obtained in SNr between the two actions (Fig. 6B, black and copper lines in middle panel), but at one point both will lead to completely inhibited populations in BG output nuclei, resulting in dual selection. Such a selection agent would work over a very limited dynamic range, and action selection would be possible only during a narrow window (Fig. 6B, middle panel, blue line). Instead, the contrast should preferably be obtained over a larger interval (Fig. $6 B$, bottom panel) with a mechanism ensuring that the contrast between the two actions is maintained over a larger range of inputs. In Humphries et al. (2006), the size of the activated MSN pool was not considered. Here we also propose that action selection should work over different proportions of activated MSNs. Thus it is important for the action selection agent to be able to select and gate through only one of the simulated cortical input states over a range of different input strengths and for various numbers of active presynaptic neurons.

To test the performance of action selection in our model, we measured the difference in firing rates between two groups of SNr neurons (action pools). We then generated a figure with the difference in firing rates between two action pools in $\mathrm{SNr}$ by presenting the network with a combination of cortical inputs leading to the activation with different strengths of striatal populations linked to actions 1 and 2 (Fig. 6C). Selection status was illustrated for each data point as a pie chart with no selection (white), selection action 1 (blue), selection action 2 (red), or dual selection (black). For each data point, we ran several simulations, and the proportions of outcomes are illus- 
A

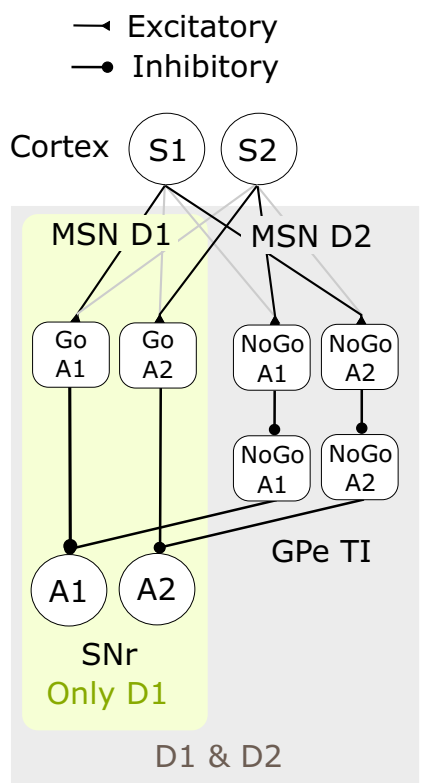

B
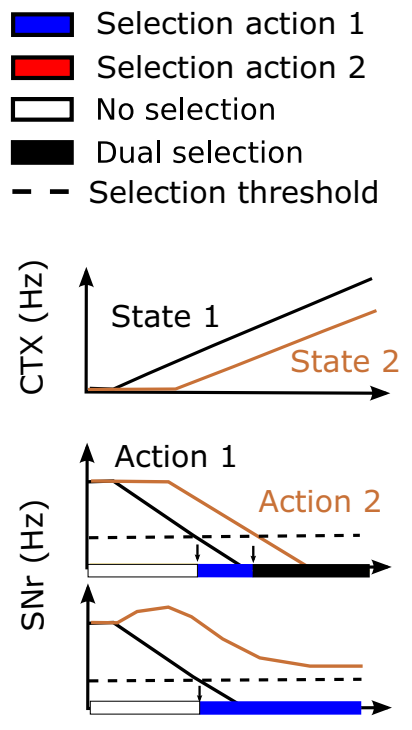

Simulation trial
C

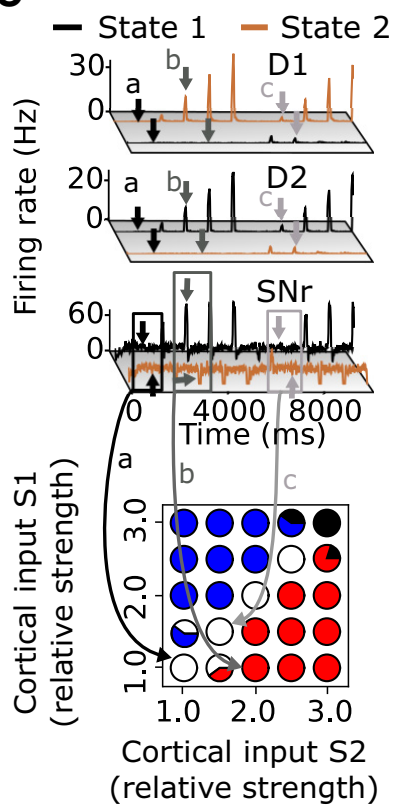

Figure 6. Illustration of the action selection network tested. A, Illustration of a simplified action selection network with two action channels. Light gray lines from cortex to MSNs indicate that these connections are weak (e.g., due to learning), whereas black indicates strong connections. Cortical state S1 connects to one striatal population in the direct (Go) pathway (denoted Go_A1), which inhibits the action 1 (A1) population in SNr. S1 also activates a striatal population in the indirect (NoGo) pathway (denoted NoGo_A2), hypothesized to disinhibit SNr populations not compatible with action 1 (i.e. in this example action 2), and vice versa for cortical state S2. Green box indicates the network in which only the direct (Go) pathway is activated, and the larger gray box indicates the network in which both the direct (Go) and indirect (NoGo) pathways are activated. SNr A1 and A2 populations are in turn assumed to control targets in the brain stem and/or thalamus via disinhibition. B, Illustration of two types of action selection scenarios. Top graph shows the firing rates of two hypothesized cortical states, state 1 (black) and state 2 (copper) that have been associated with two different actions after learning. Bottom two graphs show the resulting SNr firing rates for the co-activation of S1 and S2 representing action 1 (black) and 2 (copper). White indicates the interval on the $x$ axis in which the SNr firing rates controlling action 1 and 2 are both above the selection threshold (i.e., no selection); blue indicates the interval in which the firing rate for action 1 is below the selection threshold, but action 2 is not (i.e., selection of action 1); and black indicates the interval in which both actions are selected (dual selection). In the beginning, good contrast can be obtained in SNr between the two actions (black and copper lines in middle panel), but at one point both will lead to completely inhibited populations in BG output nuclei, resulting in dual selection. Such a selection agent would work over a very limited range of inputs, and action selection would be possible only during a narrow window (middle panel, blue line). Instead, the capability to select only one action should preferably be obtained over a larger interval (bottom panel) with a mechanism ensuring that the contrast between the two actions is maintained over a larger range of inputs. C, Illustration of how assumed cortical input combinations could activate striatal populations, in turn facilitating action 1 and action 2 , and how illustrations of action selection outcomes are made. First and second panels show examples how D1 (Go) or D2 (NoGo) populations are activated for consecutive trials by cortical state 1 and 2. For example, state 2 are stepwise increased over five trials and state 1 is kept constant, and this is then repeated, e.g., five times but with state 1 increased each time. Third panel shows the resulting firing rate of SNr neurons representing action 1 (black) and action 2 (copper). Black, gray, and light-gray boxes are three examples of input combinations, a, b, c, as illustrated with the arrows and corresponding letters. Bottom panel illustrates how these types of simulations are classified into a selection pie chart. In each pie (circle) the relative proportions of action selection outcomes are represented with the respective colors: white for no selection, black for dual selection, blue for selection of action 1, and red for selection of action 2.

trated in each pie chart. The concept of selection threshold was adopted from Humphries et al. (2006), in which selection is considered to have occurred when firing rate becomes sufficiently low in SNr. Here the threshold is defined as $50 \%$ below base firing rate of SNr neurons (see Methods).

The indirect pathway is important for improving the dynamic range in the output, SNr, in response to cortical inputs to MSNs. The hypothesis that BG are involved in the selection of actions has been around for at least 20 years (Mink, 1996), but it is still debated to what extent BG are indeed involved (Nambu, 2008). Computational models have successfully been used to show that the idea is feasible (Gurney et al., 2001a; Humphries et al., 2006). These studies have made significant progress, stressing that the action selection hypothesis needs to be taken seriously. Here we have the chance to further test the action selection hypothesis within our quite quantitative model of the BG. The model has been built based on data collected from the literature with regard to activity levels, synaptic connectivity, and dopamine effects and validated based on known dynamics (i.e., not function) 
A
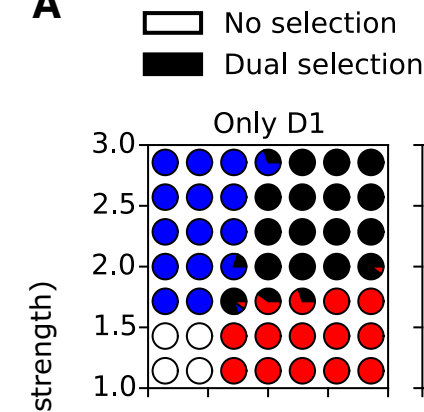

No MSN $\rightarrow$ MSN

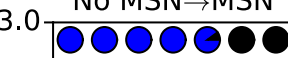

$2.5-000000$ 000000

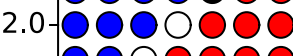

$1.5-00000000$

1.00000000

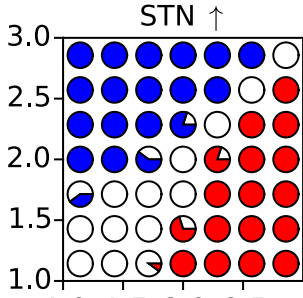

$\begin{array}{llll}1.0 & 1.5 & 2.0 & 2.5\end{array}$

Cortical input state $\square$ Action 1

D1 \& D2
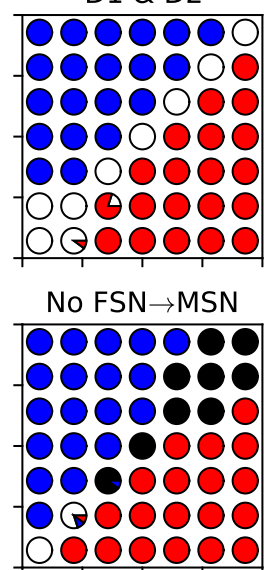

$\mathrm{STN} \uparrow$ no TA $\rightarrow$ MSN

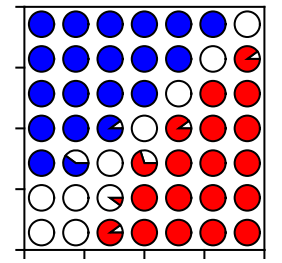

$\begin{array}{lllll}1.0 & 1.5 & 2.0 & 2.5 & 3.0\end{array}$
B
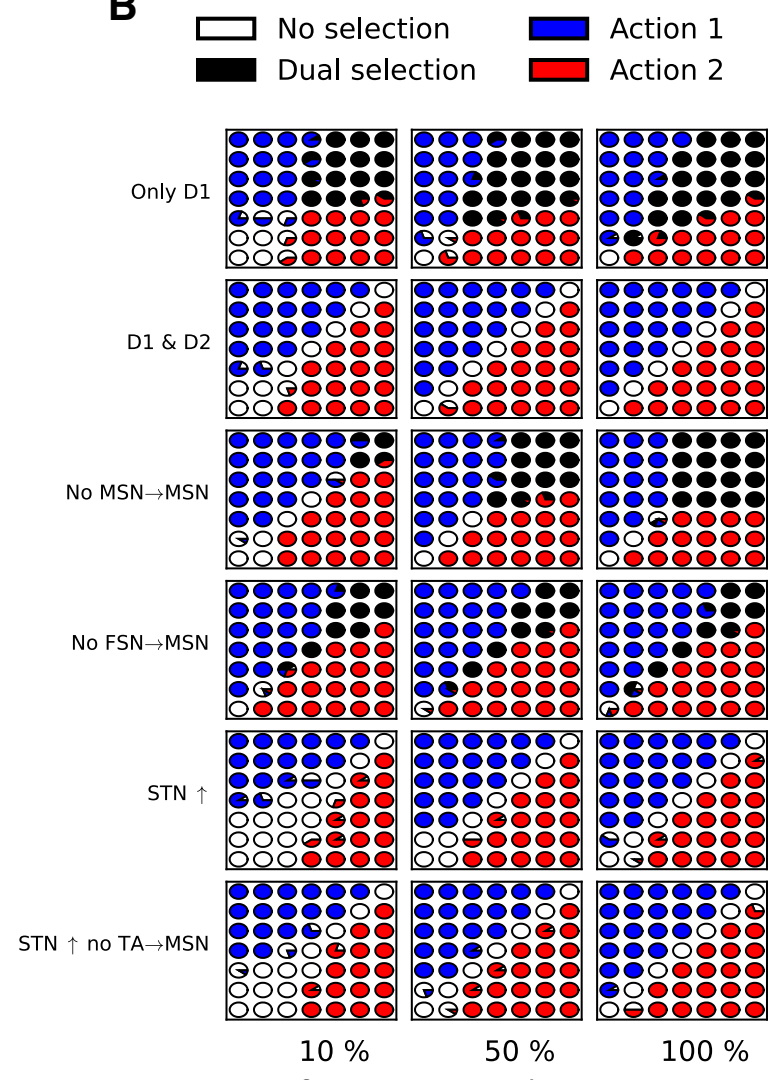

Proportion of MSNs activated in an action poo

Figure 7. Action selection performance of the basal ganglia network. $\boldsymbol{A}$, Top left panel shows the action selection outcome with only the direct pathway activated, with respectively cortical input (i.e., S1 or S2) from $1 \times$ the base level to $3 \times$ the base level leading to selection of actions 1 and 2. Top right panel shows the action selection when both direct and indirect pathways are activated (similar action inputs as in top left panel). Middle left panel shows the action selection when both direct pathways are activated and MSN collaterals have been removed (similar inputs as in top right panel). Middle right panel shows the result when both direct and indirect pathway are activated and FSN-MSN inhibition has been removed (similar action inputs as in top right panel). Bottom left panel shows the action selection when both direct and indirect pathways are activated and in addition the hyperdirect pathway is co-activated through a burst in STN (similar action inputs as in top right panel). Bottom right panel shows the outcome when direct, indirect, and hyperdirect pathways are activated and GPe TA-MSN inhibition has been removed. For all six plots, $20 \%$ of the total MSN D1 or D2 pools were activated (the maximal MSN D1 or D2 action pool equaled each half of the total striatal MSNs). B, Action selection performance when scaling the size of the activated MSN pool. First row shows the action selection with only direct pathway activated, same as top left figure in $\boldsymbol{A}$, but varying the relative size of the activated MSN population between $10 \%$ and $100 \%$. Second row shows the same as the first row, except for the scenario when both direct and indirect pathways are activated. Third and fourth rows show the result for the scenario when both direct and indirect pathways are activated (as in row 2) and when in addition MSN collaterals or the FSN-MSN inhibition is removed. Fifth and sixth rows show the effect when the hyperdirect pathway is activated during action selection with and without TA-MSN present.

in the control and dopamine-depleted state; thus there is nothing that says that it should work as an action selection network. To test action selection capability, we started out with a model version with assumed action channels only in the direct pathway. It can be seen that the network performs action selection over a range of inputs (Fig. $7 A$, top left panel) but degenerates at higher inputs (black area), resulting in dual selection. Thus with only the direct pathway present, action selection can be performed, but only over a limited range of inputs. We then wanted to see what happened if we included the indirect pathway. Would it improve action selection such that dual selection is less likely? Indeed it did: adding action channels in the indirect pathway improves the contrast at higher input (Fig. 7A, top right panel). Finally we were interested in whether the model would perform action selection when varying the total size of the MSN pool assumed to be representing the action channel populations. In Fig. 7A, the D1 and D2 populations represent $20 \%$ of the total MSNs in the network, but interestingly, the effect persists when varying the number of active D1 and D2 neurons included into the action pools between $10 \%$ and $100 \%$ (Fig. 7B). Thus we found that the model supported the idea that $B G$ can perform action selection, and that the indirect pathway is there to increase the dynamic range of the system by enabling action selection over a larger range of competing inputs. 


\section{Inhibitory inputs to MSNs differentially affect action selection}

Action selection deteriorates when MSN collaterals and/or feed-forward striatal inhibition are removed. Bronfeld et al. (2011) observed that when GABA-A is inhibited with bicuculline in the striatum, monkeys started to produce coarser and repetitive movements. They termed this loss of specificity (LOS). We were now interested in whether the model could give an explanation why inhibiting GABA-A produced LOS. To test the role of inhibition, we blocked GABA synaptic projections, one type at a time, from MSN collaterals and FSN. We found that when removing the inhibition onto MSNs from either collaterals or the FSNs, the model showed evidence of LOS. For both scenarios, action selection gets worse with dual selection when combining two strong inputs (Fig. 7A, middle panels). The model thus predicts that both MSN collaterals and FSNs are important for robust action selection in BG. This observation also holds when a different proportion of the MSN pool is activated (Fig. 7B).

\section{STN as a transient stop signal}

STN, through the cortical hyperdirect pathway, is proposed to act as a stop signal (Gillies and Willshaw, 1998; Frank, 2006), giving striatum and GPe enough time to resolve high conflict in action selection situations. Experiments also suggest that STN is involved in the cancellation of already initiated motor responses (Eagle and Robbins, 2003; Eagle et al., 2008; Schmidt et al., 2013, Mallet et al. 2016). We ran simulations in which we added input to STN as a 100-ms pulse (to match the duration of the striatal activation seen in experiments) in parallel with activation of the striatal action pools (Fig. $7 \mathrm{~A}$, bottom left panel). We observed that action selection at low cortical input was stopped or delayed, in line with the claim that STN can be responsible for a transient stop signal in the BG (see also Lindahl et al., 2013). When GPe TA projections to MSNs were removed, the stop signaling via STN was weakened. This is in agreement with suggestions made in Mallet et al. (2016) that the activity in MSNs could be decreased by activity in the TA-MSN inhibitory synapses (also compare Fig. 7B, bottom two panels).

\section{Action selection in the dopamine-depleted network}

Action selection deteriorates when dopamine is removed from the network. Dopamine loss leads to increase in synchrony and oscillations in BG as seen above, but it is not clear whether dopamine loss would also affect action selection in our model. Actually the pathophysiology of PD in BG is still not well understood (Nambu, 2008). The firing rate model by DeLong (1990) proposed that imbalance between the direct and indirect pathways changes the mean firing rate of BG output nuclei and induces PD. The firing pattern model by Bergman et al. (1998) instead suggests that it is the increase in oscillations that interfere with BG information processing in PD. It is not easy to resolve whether it is the firing rate or firing pattern model that better explains the pathophysiology behind PD. The answer is probably both. We wanted to see whether our model could shed light on this controversy. Without dopamine, the model network is not able to efficiently perform action selection (Fig. 8A, top second panel). Dopamine changes the balance between the direct and indirect pathways, such that the indirect pathway becomes more dominant, leading to action selection failure and an increased firing rate in the $\mathrm{SNr}$. Thus the model supports the assumption that excessive firing in the output of BG can underlie some of the symptoms in PD.

\section{Reversal of selected dopamine-depletion induced network changes restores action selection}

Dopamine depletion induced action selection deterioration, but this can be restored in the model by reverting the dopamine-induced change in mainly CTX-MSN D1 and CTX-MSN D2 synapses (Fig. 8A), despite still having synchrony or oscillations present (compare Fig. 4).

\section{Effects of synaptic increases or decreases on oscillations and BG action selection capability}

Several perturbations to the connections in BG lead to a decrease in oscillations, and in some cases also improved action selection, thus making them potential targets for animal models or translational studies. For example, in Fig. 4, it was shown that reverting just one parameter at a time had effects on the synchronizations and oscillations in the dopamine-depleted network. To further test how parameter perturbations could affect both oscillations and action selection, we set up simulations in which, on top of beta oscillations from cortex in the lesioned model, we added the test of action selection. Action selections were measured as a certain decrease in mean firing rate of SNr neurons during the action selection phase (see Methods). It was found that decreasing or increasing the synaptic efficacy in approximately half (11/ 25 ) of the modeled connections led to a decrease in synchrony or oscillations, and that in a subset of them the change (i.e., increased connectivity for TA-MSN D2 and MSN D2-MSN D2 and removing connectivity for MSN D2-TI) also improved action selection ability. Table 10 lists the connections with either decreased or increased efficacy that in Fig. $8 B$ show significant reduction of synchrony or oscillations in several nuclei. All manipulations of connections in Table 10 could thus serve as potential targets for animal models or translational studies. Especially interesting are the manipulations that also improve action selection (Fig. 8D).

\section{Effects of nuclei lesion and stimulation on oscillations and BG action selection capability}

By silencing or increasing the activity of specific BG nuclei, an improvement in action selection is achieved (Table 11 and Fig. 8C, D). Lesioning GPe and STN decreases both the synchrony and oscillations in several nuclei in the network, lesioning is especially effective in STN (Fig. 8C). Both predictions are in accordance with experiments. Lesion therapies targeting GPe, GPi, and STN have successfully been used to alleviate PD symptoms (Okun and Vitek, 2004). It can also be seen that increases in the activity in STN leads to a decrease in 


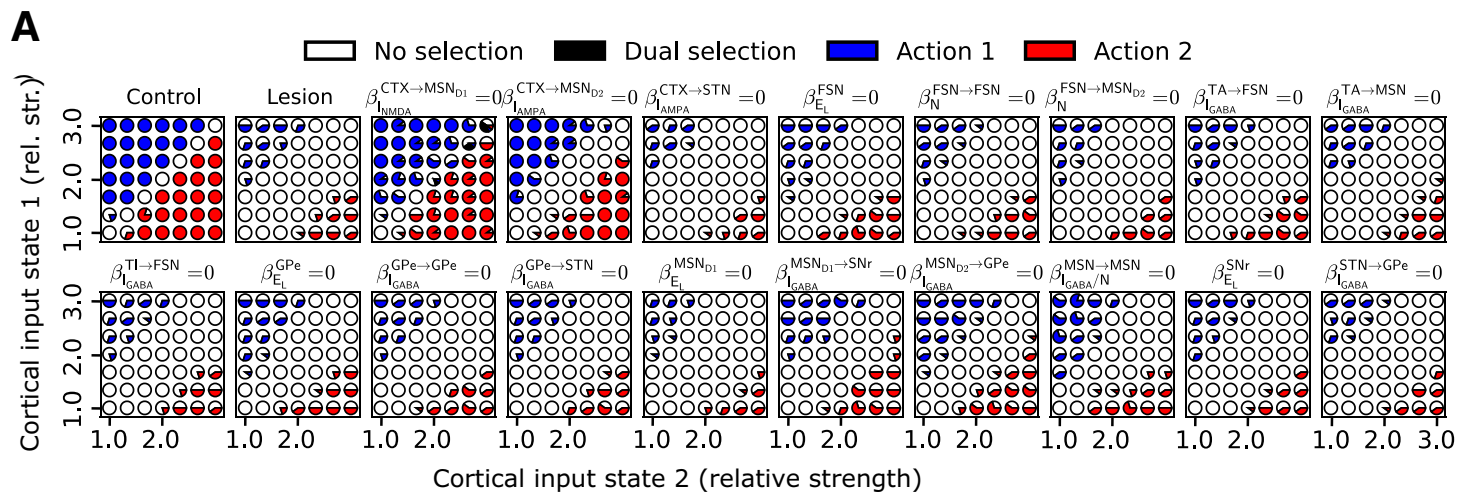

B

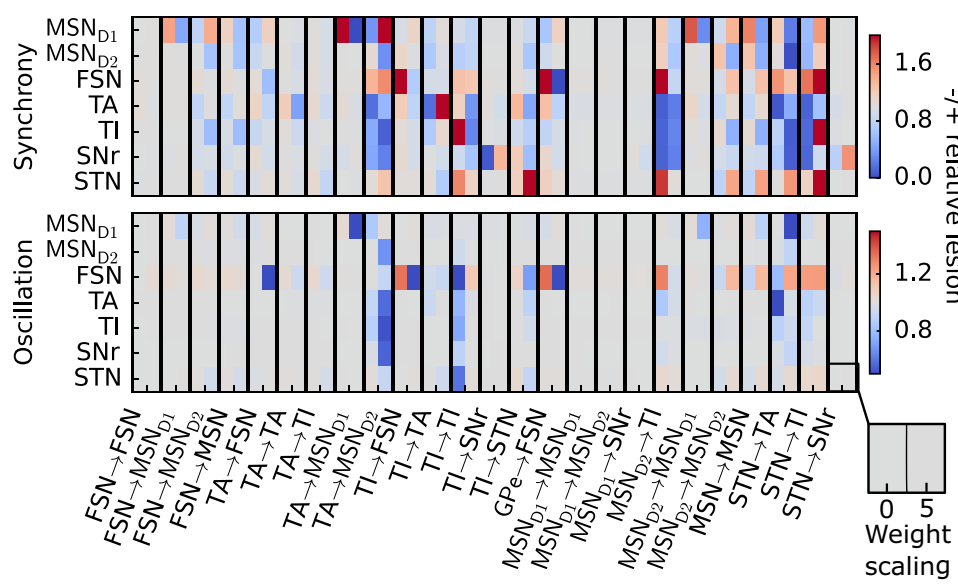

C

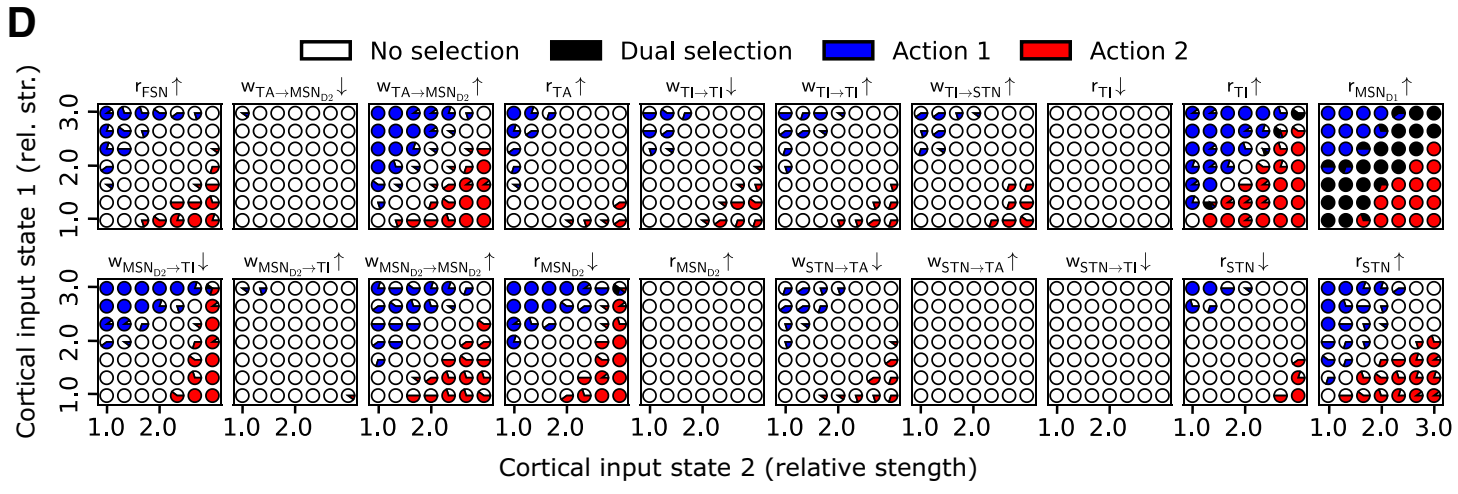

Figure 8. Restoring network dynamics and function after dopamine lesion. A, Action selection performance of control and lesioned networks. Left upper panel shows an action selection heat map of the control network with direct and indirect pathways both activated. Second upper panel shows the same but for the lesioned network. The remaining panels show the result for the lesioned network but with the dopamine parameter displayed in the heading restored to the control network parameter value. (compare with Fig. 4B). B, Change in synchrony and oscillations in MSN D1, MSN D2, FSN, GPe TA, GPe TI, SNr, and STN relative to the lesioned network model when silencing/increasing each connection. $\boldsymbol{C}$, Change in synchrony and oscillations in MSN D1, MSN D2, FSN, GPe TA, GPe TI, SNr, and STN relative to lesioned network model when inhibiting/exciting each nucleus separately. A current was injected with a negative or positive value in each nucleus. The hyperpolarizing and depolarizing current steps were equally large, where the amplitude of the hyperpolarizing current was adjusted to silence the specific nucleus (mimicking a lesion of the nucleus). $\boldsymbol{D}$, Action selection performance for a number of manipulations either to neuron excitability or to synaptic efficacy.

synchrony and oscillations, which is again in line with what deep brain stimulation (DBS) in STN has proven to be an effective method for relieving PD symptoms.

The model holds one possible answer to the paradox that either decreased activity in STN (by lesioning it) or increased activity in STN (through high-frequency stimu- lation) can reduce pathological dynamics. When STN is lesioned in the model, TA and TI neurons dramatically decrease in firing rate and no longer convey oscillations to $\mathrm{SNr}$ neurons. On the other hand, when STN firing rate is increased, TA firing rate is elevated, in turn reducing MSN firing rate. This shifts the balance between oscillatory 
Table 10. Effect of connection on synchrony (+, increase; -, decrease, 0 , no change), oscillations $(+/-/ 0)$, and action selection

\begin{tabular}{|c|c|c|c|c|c|c|}
\hline Connection & Scaling & TA & $\mathrm{TI}$ & $\mathrm{SNr}$ & STN & Action selection \\
\hline TA-MSN D2 & 0 & $+/ 0$ & $+/ 0$ & $+/ 0$ & $0 / 0$ & Worse \\
\hline TA-MSN D2 & 5 & $+/+$ & $+/+$ & $+/+$ & $0 / 0$ & Improved \\
\hline TI-TI & 0 & $0 /+$ & $-/+$ & $0 /+$ & $-1+$ & None \\
\hline TI- TI & 5 & $+/ 0$ & $+/ 0$ & $+/ 0$ & $0 / 0$ & None \\
\hline TI-STN & 5 & $+/ 0$ & $+/ 0$ & $+/ 0$ & $-/ 0$ & None \\
\hline MSN D2-TI & 0 & $+/+$ & $+/ 0$ & $+/ 0$ & $-/ 0$ & Improved \\
\hline MSN D2-TI & 5 & $+/ 0$ & $+/ 0$ & $+/ 0$ & $0 / 0$ & Worse \\
\hline MSN D2-MSN D2 & 5 & 0/0 & $+/ 0$ & $0 / 0$ & $-/ 0$ & Improved \\
\hline STN-TA & 0 & $+/+$ & $0 / 0$ & $0 / 0$ & $0 / 0$ & None \\
\hline STN-TA & 5 & $+/ 0$ & $+/ 0$ & $+/ 0$ & $-/ 0$ & Worse \\
\hline STN-TI & 0 & $+/ 0$ & $+/ 0$ & $+/ 0$ & $0 / 0$ & Worse \\
\hline
\end{tabular}

inputs from cortex conveyed via MSN D2 and STN onto TI neurons (compare Fig. 4C).

Both decreasing and increasing MSN D2 activity counteracts synchrony and oscillations in GPe and SNr. Loss of dopamine leads to increasing activity in MSN D2 neurons. Decreasing MSN D2 activity (Fig. $8 C$ ) proves to be an effective way to annihilate synchrony and oscillations in GPe and SNr. On the other hand, increasing MSN D2 activity silences $\mathrm{GPe}$, resulting in the same output as lesioning GPe. However, only the decrease in MSN D2 activity is predicted to improve action selection capability (Fig. 8D).

\section{Model robustness: some observations}

The BG model was built on current data and knowledge, but several parameters are unknown, especially with regard to the synaptic connectivity patterns between different nuclei. During the process of validating the model against Mallet et al (2008) additional insights were received regarding parameter constraints. These findings are described below.

One observation was that synaptic dynamics of TA to MSN could control oscillations in striatum. The dynamics of the GABAergic TA-MSN synapse prevents oscillations in striatum under normal dopamine conditions. The time constant that Glajch et al. (2016) recorded for TA synapses on MSNs is $>6$ times longer than a normal GABA synapse (10 ms vs. $60-90 \mathrm{~ms})$. Is there a reason this is important? It turned out that this time constant was not crucial in explaining Mallet et al. (2008), but instead oscil-

Table 11. Effect of nucleus firing rate on synchrony $(+$, increase; -, decrease; 0 , no change), oscillations (+/-/0), and action selection

\begin{tabular}{lllllll}
\hline Nucleus & Firing rate & $\mathrm{TA}$ & $\mathrm{TI}$ & $\mathrm{SNr}$ & $\mathrm{STN}$ & Action selection \\
FSN & Increase & $+/ 0$ & $+/ 0$ & $+/+$ & $-/ 0$ & Improved \\
$\mathrm{TA}$ & Increase & $+/+$ & $+/+$ & $+/+$ & $-/-$ & Worse \\
$\mathrm{TI}$ & Silent & $+/+$ & $+/+$ & $+/ 0$ & $-/ 0$ & Worse \\
TI & Increase & $+/+$ & $-/ 0$ & $+/+$ & $-/-$ & Improved \\
MSN D1 & Increase & $0 / 0$ & $0 / 0$ & $+/+$ & $0 / 0$ & Improved \\
MSN D2 & Silent & $+/+$ & $+/+$ & $+/+$ & $-/-$ & Improved \\
MSN D2 & Increase & $+/+$ & $+/+$ & $+/+$ & $-/-$ & Worse \\
STN & Silent & $+/+$ & $+/+$ & $+/+$ & $+/+$ & Worse \\
STN & Increase & $+/ 0$ & $+/+$ & $+/ 0$ & $+/+$ & Improved
\end{tabular}

lations in the MSN populations started to occur in the control model with normal dopamine levels when the time constant was decreased too much (Fig. 9A). Thus the simulations predict that the time constant of the TA-MSN synapse indeed needs to be significantly slower than the other GABA synapses to prevent oscillations in MSNs.

A second insight gained was that MSN-TA connectivity can affect the phase relation and even can reverse the phase relation between $\mathrm{TI}$ and TA in the model with dopamine depletion. Chuhma et al. (2011) showed that there is a population of GPe neurons that do not receive input from MSN neurons. They showed that $28 \%$ of the GPe neurons in their study were not activated by light stimulation of striatal fibers. In the current study, we assumed that TA neurons corresponded to this population. One reason was that it made sense, since TA neurons fire in phase with STN, implying that they are controlled by excitatory input from STN rather than inhibitory inputs from MSNs. We wanted to challenge this assumption. MSN-TA synapses were added to the model with conductance similar to that of MSN-TI synapses. The model shows that even with an MSN-TA fan-in (number of incoming of connections) at 25 (compared with MSN-TI at 500 ), the phase relation of $\mathrm{TI}$ and TA was significantly altered (Fig. 9B). With fan-in at 100, the phase relation starts to reverse where $\mathrm{TI}$ and TA neurons now are in phase with each other. Thus the model supports the assumption that TA indeed receives none or only a low number of connections from MSNs.

A third insight was that the synaptic delay between cortex and striatum/STN is important. Synaptic delays between cortex and striatum/STN affect the phase relation between STN and TI neurons. Cortical oscillations can affect TA neurons though two main pathways, one inhibitory through cortex to MSNs and one excitatory through cortex and STN. Mallet et al. (2008) showed that STN and TI neurons fire out of phase in lesioned rats. It was crucial that the model could reproduce this dynamic, and we hypothesized that the delay between cortex and striatum/STN could play a crucial role in enabling us to reproduce the dynamic. We found that stepwise increasing the delay from 2.5 up to $20 \mathrm{~ms}$ for cortex to striatum could distort the phase relation between STN and TI neurons (Fig. 9C), and the same holds for cortex/STN (Fig. $9 D$ ). Thus the phase relation of STN and TI neurons is likely governed by the corticostriatal/STN delays, and this suggests that these parameters need to be controlled well in the real system.

We also tested the role of increased connectivity between STN and GPe for TI-TA phase relation. The model does not support stronger GPe-STN synapses than those we chose to have. Farries et al. (2010) observed that when removing GPe connections to STN, the firing rate of STN neurons doubles and the cortical input to STN and GPeSTN conductance were originally tuned to reproduce this result (compare Lindahl et al., 2013). However, another study (Féger and Robledo, 1991) reported that STN firing rate increases fivefold when removing input to STN. Moreover, Terman et al. (2002) showed that strengthening of the GPe-STN synapses leads to oscillations in GPe and 
A
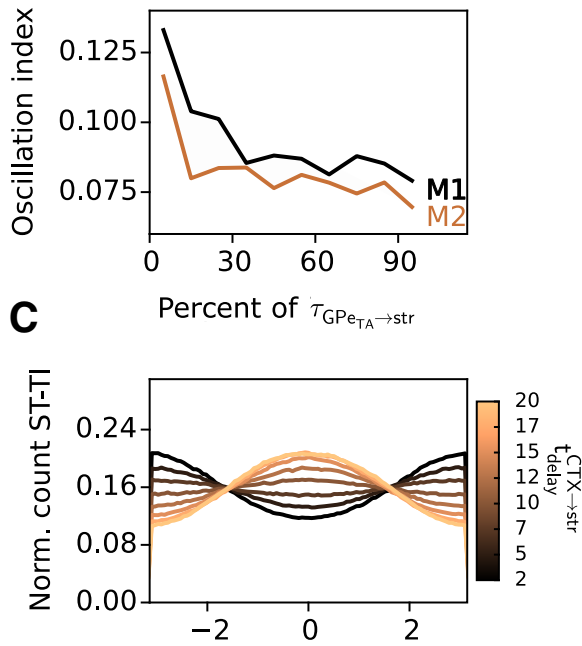

E

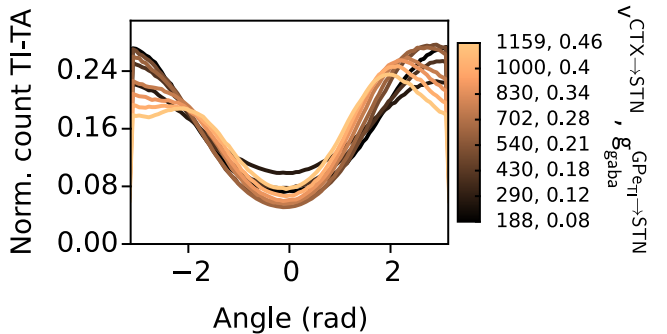

B
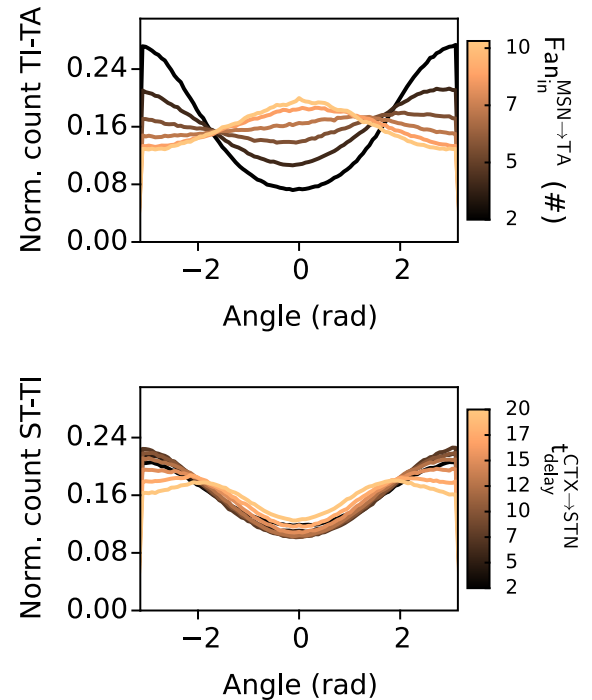

F

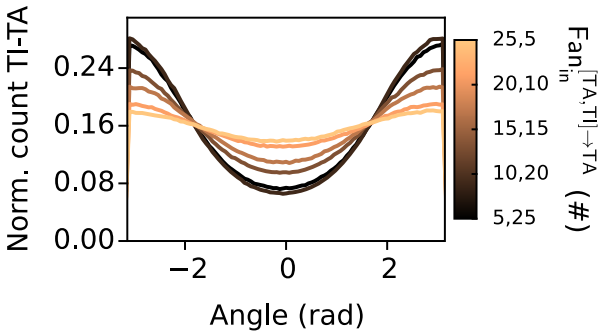

Figure 9. Effect of network parameter perturbations. $\boldsymbol{A}$, Effect of synaptic decay $\tau_{G P e_{T \rightarrow} \rightarrow \text { striatum }}$ on oscillations in MSNs in the control network. Black line shows effect on MSN D1 and copper line shows effect on MSN D2. $\boldsymbol{B}$, Phase relation between TI and TA neurons when fan-in to TA neurons from MSN was varied between 25 and 125 with step 25. $\boldsymbol{C}$, Phase relation between STN and TI neurons when varying the delay between cortex and striatum from 2.5 to $20 \mathrm{~ms}$ with steps of $2.5 \mathrm{~ms}$. D. Phase relation between STN and TI neurons when varying the synaptic delay between cortex and STN from 2.5 to $20 \mathrm{~ms}$ with steps of 2.5 ms. $\boldsymbol{E}$, Phase relation between TI and TA neurons when keeping STN activity constant by varying the synaptic weight between TI and STN in parallel with adjusting the background input rate from cortex to STN. $\boldsymbol{F}$, Phase relation between TI and TA neurons when fan-in to TA neurons from TA and TI neurons were, respectively, [25,5], [20,10], [15,15], [10,20], and [5,25].

STN neurons. Thus we hypothesized that GPe-STN synapses were stronger. Therefore we modified GPe-STN synapses and input to STN neurons such that removing GPe neurons increased STN firing rate from 20 up to 50 $\mathrm{Hz}$ with $5-\mathrm{Hz}$ steps. We found that strengthening $\mathrm{GPe}-$ STN could not be supported by the model, since it introduces a double peak in the phase relation of TI and TA neurons (Fig. 9E). Additionally (not shown), the firing rate of $\mathrm{TI}$ and $\mathrm{TA}$ in the lesioned model increases a lot and no longer compares to Mallet et al. (2008).

The model also suggests that the input from $\mathrm{TI}$ and $\mathrm{TA}$ onto TA preferably comes from TI neurons rather than TA neurons. It is known that $\mathrm{GPe}$ neurons receive collateral input from surrounding GPe neurons. What is not known is how TI and TA neurons connect to each other. Because TI neurons send significantly more collaterals than TA neurons (Mallet et al., 2012) and TI neurons are five times more numerous, then by share numbers TI neurons should receive most of their collaterals from TI neurons. A similar argument could be made for TA neurons; that is, they should receive most of the collaterals from TI. But this may not be true, so we ran five simulations in which the fan-in from TI and TA neurons to TA neurons was altered. The phase relation between TI and TA neurons is weakest when the majority of inputs to TA are from TA and strongest when the majority comes from TI neurons (Fig. $9 F$ ). Thus the model predicts that TA neurons preferably receive input from other $\mathrm{TI}$ neurons.

\section{Discussion}

We investigate in a spiking network model of BG the mechanisms for both the control of oscillations and spike synchronization during dopamine-depleted stages and further correlate the network dynamics to how action selection is supported. We explore how different sources of inhibition in striatum contribute to these network phenomena and hypothesized function. Our model was built using parameters compiled from a large set of experimental data sets, and the subsequent validation of the model against multiple experimental observations aims at making it a quantitative model of the BG system that can be used to make predictions and help increase our understanding of the mechanisms behind action selection and dynamic features occurring during PD, and in the future 
function as a framework for incorporating biophysically detailed model modules of various neuron types or even whole nuclei. During the model building and model validation process, we explored the effect of several model parameters. We predict that inhibition from FSN and GPe in striatum is relatively more important during low cortical input, whereas the collateral MSN network becomes more significant during higher cortical input. It is also demonstrated by implanting a topology in the weak collateral MSN network that the contrast between input signals can be enhanced, which could facilitate the action selection capability. The indirect pathway is predicted to increase the dynamic range of action selection signaling in the BG, where action selection is preserved also for stronger cortical inputs, a result in line with Humphries et al. (2006). The feed-forward and collateral inhibition in the striatum is also predicted to be important for increasing the dynamic range. It is also demonstrated that a brief, high input from STN can stop or delay action selection, similar to what Frank (2006) predicts, and here the GPe TA to MSN inhibition contributes in line with Mallet et al. (2016). Dopamine depletion in the network leads to an increase in spike synchrony and oscillations, and at the same time an impairment in action selection capability is seen. Our simulations predict that an important mechanism behind these changes in network dynamics and $B G$ function is the increase in the activation of the CTX-MSN D2 pathway, the weakening in the MSN collateral inhibitory network, and to some extent the decreased excitability of $\mathrm{GPe}$ neurons after dopamine depletion. It is shown that when successively manipulating the excitability in each of the BG nuclei, either increasing or decreasing their excitability, a reduction in the synchronization and the oscillations as well as an improvement in action selection can be achieved. We also found that either a decrease or an increase in the connectivity of at least 10 different connections in the BG network can decrease the synchrony and oscillations in the dopamine-depleted state. For about half of these changes in the connectivity the action selection capability is improved.

The effective connectivity used in this model compares well to the predicted connectivity from a rate model by Nevado-Holgado et al. (2014) that was fitted to part of the same data set. They similarly predicted that the connectivity between MSN-GPe TI is strong, whereas MSN-GPe TA projections are weak. It is also shown here that TI-TA should be stronger than TA-TA, thus implying that TI neurons have a strong control over TA neurons. Both these findings also seem to be supported by experiments (Mallet et al., 2012). However Nevado-Holgado also predicted that STN connectivity is stronger to TA than to TI. This is something we did not find; actually the conductance of STN-TA synapses in our model is $\sim 3$ times weaker than STN-GPe TI connections. However, we still saw that STN influences the activity in GPe TA in practice, since the STN input constitutes a larger proportion of the total excitatory input to GPe TA compared with the total excitatory input to GPe TI. Thus the resulting effective connectivity in our spiking network model is still in line with the predicted (relative) connectivity used in the rate model by Nevado-Holgado et al. (2014).

BG modeling has made significant progress in proposing how the architecture of BG supports the hypothesis that $B G$ are a general-purpose action selection device (Gurney et al., 2001a,b; Humphries et al., 2006). For example, the spiking network model of Humphries et al. (2006) contained detailed BG physiology and could replicate multiple experimental data sets. Since then, new data on synapses and connectivity have been collected and need to be included in BG models. Thus in this study, we extended the functional connectivity of previous models (e.g., Humphries et al., 2006) with short-term plasticity, used a more detailed striatal network, took into account the relative BG nuclei sizes, and also included the GPe TA/TI populations. Similar to Humphries et al. (2006), we found that BG can support an action selection function.

Our spiking BG network model provides novel insights about action selection, synchrony, and oscillation-quenching mechanisms and provides a framework for investigating PD and other BG-related diseases. The current model, however, lacks the thalamic and cortical feedback loops from the BG output nuclei to influence the BG input via one longer thalamus-cortex-striatal loop and a shorter thalamus-striatal loop (Smith et al., 2010). Spike synchrony, network oscillations, and BG action selection functionality could very well be affected by these loops. Although implementing the longer loop via cortex would be a major project, including the shorter loop would be a natural next step.

\section{References}

Abbott LF, Regehr WG (2004) Synaptic computation. Nature 431: 796-803. CrossRef Medline

Abbott LF, Varela J a, Sen K, Nelson SB (1997) Synaptic depression and cortical gain control. Science 275:220-224. Medline

Abdi A, Mallet N, Mohamed FY, Sharott A, Dodson PD, Nakamura KC, Suri S, Avery S V, Larvin JT, Garas FN, Garas SN, Vinciati F, Morin S, Bezard E, Baufreton J, Magill PJ (2015) Prototypic and arkypallidal neurons in the dopamine-intact external globus pallidus. J Neurosci 35:6667-6688. CrossRef Medline

Ammari R, Lopez C, Bioulac B, Garcia L, Hammond C (2010) Subthalamic nucleus evokes similar long lasting glutamatergic excitations in pallidal, entopeduncular and nigral neurons in the basal ganglia slice. Neuroscience 166:808-818. CrossRef Medline

Atherton JF, Bevan MD (2005) Ionic mechanisms underlying autonomous action potential generation in the somata and dendrites of GABAergic substantia nigra pars reticulata neurons in vitro. $J$ Neurosci 25:8272-8281. CrossRef Medline

Baufreton J, Atherton JF, Surmeier DJ, Bevan MD (2005) Enhancement of excitatory synaptic integration by GABAergic inhibition in the subthalamic nucleus. J Neurosci 25:8505-8517. CrossRef Medline

Baufreton J, Bevan MD (2008) D2-like dopamine receptor-mediated modulation of activity-dependent plasticity at GABAergic synapses in the subthalamic nucleus. J Physiol 586:2121-2142. CrossRef Medline

Baufreton J, Kirkham E, Atherton JF, Menard A, Magill PJ, Bolam JP, Bevan MD (2009) Sparse but selective and potent synaptic transmission from the globus pallidus to the subthalamic nucleus. $J$ Neurophysiol 102:532-545. CrossRef Medline

Bergman H, Feingold A, Nini A, Raz A, Slovin H, Abeles M, Vaadia E (1998) Physiological aspects of information processing in the basal 
ganglia of normal and parkinsonian primates. Trends Neurosci 21:32-38. Medline

Berke JD, Okatan M, Skurski J, Eichenbaum HB (2004) Oscillatory entrainment of striatal neurons in freely moving rats. Neuron 43: 883-896. CrossRef Medline

Berthet P, Lindahl M, Tully P, Hellgren Kotaleski J, Lansner A (2016) Functional relevance of different basal ganglia pathways investigated in a spiking model with reward dependent plasticity. Front Neural Circuits 10:53. CrossRef

Bevan MD, Booth P, Eaton S (1998) Selective innervation of neostriatal interneurons by a subclass of neuron in the globus pallidus of the rat. J Neurosci 18:9438-9452. Medline

Bevan MD, Wilson CJ (1999) Mechanisms underlying spontaneous oscillation and rhythmic firing in rat subthalamic neurons. J Neurosci 19:7617-7628.

Bevan MD, Wilson CJ, Bolam JP, Magill PJ (2000) Equilibrium potential of $\operatorname{GABA}(\mathrm{A})$ current and implications for rebound burst firing in rat subthalamic neurons in vitro. J Neurophysiol 83:3169-3172. Medline

Bracci E, Centonze D, Bernardi G, Calabresi P, Bracci E, Centonze D, Centonze D, Bernardi G, Bernardi G, Calabresi P, Calabresi P (2002) Dopamine excites fast-spiking interneurons in the striatum. J Neurophysiol 87:2190-2194. CrossRef Medline

Brette R, Gerstner W (2005) Adaptive exponential integrate-and-fire model as an effective description of neuronal activity. J Neurophysiol 94:3637-3642. CrossRef Medline

Bronfeld M, Bar-Gad I (2011) Loss of specificity in basal ganglia related movement disorders. Front Syst Neurosci 5:38. CrossRef Medline

Bronfeld M, Belelovsky K, Bar-Gad I (2011) Spatial and temporal properties of tic-related neuronal activity in the cortico-basal ganglia loop. J Neurosci 31:8713-8721. CrossRef Medline

Bugaysen J, Bronfeld M, Tischler H, Bar-Gad I, Korngreen A (2010) Electrophysiological characteristics of globus pallidus neurons. PLoS One 5. CrossRef

Centonze D, Gubellini P, Pisani A, Calabresi P, Bernardi G (2003) Dopamine, acetylcholine and nitric oxide systems interact to induce corticostriatal synaptic plasticity. Rev Neurosci 14:207-216. Medline

Cepeda C, Buchwald NA, Levine MS (1993) Neuromodulatory actions of dopamine in the neostriatum are dependent upon the excitatory amino acid receptor subtypes activated. Proc Natl Acad Sci U S A 90:9576-9580. Medline

Chan CS, Glajch KE, Gertler TS, Guzman JN, Mercer JN, Lewis AS, Goldberg AB, Tkatch T, Shigemoto R, Fleming SM, Chetkovich DM, Osten P, Kita H, Surmeier DJ (2011) HCN channelopathy in external globus pallidus neurons in models of Parkinson's disease. Nat Neurosci 14:85-92. CrossRef Medline

Chuhma N, Tanaka KF, Hen R, Rayport S (2011) Functional connectome of the striatal medium spiny neuron. J Neurosci 31:11831192. CrossRef Medline

Connelly W, Schulz J, Lees G (2010) Differential short-term plasticity at convergent inhibitory synapses to the substantia nigra pars reticulata. J Neurosci 30:14854-14861. CrossRef Medline

Cooper AJ, Stanford IM (2000) Electrophysiological and morphological characteristics of three subtypes of rat globus pallidus neurone in vitro. J Physiol 527Pt2:291-304. Medline

Cooper AJ, Stanford IM (2001) Dopamine D2 receptor mediated presynaptic inhibition of striatopallidal GABA(A) IPSCs in vitro. Neuropharmacology 41:62-71. Medline

Corbit VL, Whalen TC, Zitelli KT, Crilly SY, Rubin JE, Gittis AH (2016) Pallidostriatal projections promote oscillations in a dopaminedepleted biophysical network model. J Neurosci 36:5556-5571. CrossRef

Cui G, Jun SB, Jin X, Pham MD, Vogel SS, Lovinger DM, Costa RM (2013) Concurrent activation of striatal direct and indirect pathways during action initiation. Nature 494:238-242. CrossRef Medline

Damodaran S, Cressman JR, Jedrzejewski-Szmek Z, Blackwell KT (2015) Desynchronization of fast-spiking interneurons reduces -band oscillations and imbalance in firing in the dopaminedepleted striatum. J Neurosci 35:1149-1159. CrossRef Medline

Damodaran S, Evans RC, Blackwell KT (2014) Synchronized firing of fast-spiking interneurons is critical to maintain balanced firing between direct and indirect pathway neurons of the striatum. J Neurophysiol 111:836-848. CrossRef Medline

DeLong MR (1990) Primate models of movement disorders of basal ganglia origin. Trends Neurosci 13:281-285. Medline

Deschênes M (1996) A single-cell study of the axonal projections arising from the posterior intralaminar thalamic nuclei in the rat. Eur J Neurosci 8:329-343. Medline

Dittman JS, Kreitzer AC, Regehr WG (2000) Interplay between facilitation, depression, and residual calcium at three presynaptic terminals. J Neurosci 20:1374-1385. Medline

Eagle DM, Baunez C, Hutcheson DM, Lehmann O, Shah AP, Robbins TW (2008) Stop-signal reaction-time task performance: role of prefrontal cortex and subthalamic nucleus. Cereb Cortex 18:178188. CrossRef Medline

Eagle DM, Robbins TW (2003) Inhibitory control in rats performing a stop-signal reaction-time task: effects of lesions of the medial striatum and d-amphetamine. Behav Neurosci 117:1302-1317. CrossRef Medline

Ellender TJ, Huerta-Ocampo I, Deisseroth K, Capogna M, Bolam JP (2011) Differential modulation of excitatory and inhibitory striatal synaptic transmission by histamine. J Neurosci 31:15340-15351. CrossRef Medline

Farries MA, Kita H, Wilson CJ (2010) Dynamic spike threshold and zero membrane slope conductance shape the response of subthalamic neurons to cortical input. J Neurosci 30:13180-13191. CrossRef Medline

Féger J, Robledo P (1991) The effects of activation or inhibition of the subthalamic nucleus on the metabolic and electrophysiological activities within the pallidal complex and substantia nigra in the rat. Eur J Neurosci 3:947-952. CrossRef

Frank MJ (2005) Dynamic dopamine modulation in the basal ganglia: a neurocomputational account of cognitive deficits in medicated and nonmedicated parkinsonism. J Cogn Neurosci 17:51-72. CrossRef Medline

Frank MJ (2006) Hold your horses: a dynamic computational role for the subthalamic nucleus in decision making. Neural Netw 19: 1120-1136. CrossRef Medline

Freeze BS, Kravitz A V, Hammack N, Berke JD, Kreitzer AC (2013) Control of basal ganglia output by direct and indirect pathway projection neurons. J Neurosci 33:18531-18539. CrossRef Medline

Fujimoto K, Kita H (1993) Response characteristics of subthalamic neurons to the stimulation of the sensorimotor cortex in the rat. Brain Res 609:185-192. Medline

Gage GJ, Stoetzner CR, Wiltschko AB, Berke JD (2010) Selective activation of striatal fast-spiking interneurons during choice execution. Neuron 67:466-479. CrossRef Medline

Gatev P, Darbin O, Wichmann T (2006) Oscillations in the basal ganglia under normal conditions and in movement disorders. Mov Disord 21:1566-1577. CrossRef Medline

Gerfen CR, Bolam JP, Gerfern CR (2010) The neuroanatomical organization of the basal ganglia. In: Handbook of Basal Ganglia Structure and Function (Steiner H, Tseng KY, eds), pp 3-28. London: Elsevier.

Gernert M, Fedrowitz M, Wlaz P, Löscher W (2004) Subregional changes in discharge rate, pattern, and drug sensitivity of putative GABAergic nigral neurons in the kindling model of epilepsy. Eur $\mathrm{J}$ Neurosci 20:2377-2386. CrossRef Medline

Gertler TS, Chan CS, Surmeier DJ (2008) Dichotomous anatomical properties of adult striatal medium spiny neurons. J Neurosci 28:10814-10824. CrossRef Medline

Gewaltig M-O, Diesmann M (2007) NEST (NEural Simulation Tool). Scholarpedia 2:1430. CrossRef

Gillies AJ, Willshaw DJ (1998) A massively connected subthalamic nucleus leads to the generation of widespread pulses. Proc Biol Sci 265:2101-2109. CrossRef Medline 
Gittis AH, Berke JD, Bevan MD, Chan CS, Mallet N, Morrow MM, Schmidt $R$ (2014) New roles for the external globus pallidus in basal ganglia circuits and behavior. J Neurosci 34:15178-15183. CrossRef Medline

Gittis AH, Hang GB, LaDow ES, Shoenfeld LR, Atallah B V., Finkbeiner S, Kreitzer AC (2011) Rapid target-specific remodeling of fast-spiking inhibitory circuits after loss of dopamine. Neuron 71: 858-868. CrossRef Medline

Gittis AH, Nelson AB, Thwin MT, Palop JJ, Kreitzer AC (2010) Distinct roles of GABAergic interneurons in the regulation of striatal output pathways. J Neurosci 30:2223-2234. CrossRef Medline

Glajch KE, Kelver DA, Hegeman DJ, Cui Q, Xenias HS, Augustine EC, Hernández VM, Verma N, Huang TY, Luo M, Justice NJ, Chan CS (2016) Npas1 + pallidal neurons target striatal projection neurons. J Neurosci 36:5472-5488. CrossRef Medline

Gruber AJ, Solla SA, Surmeier DJ, Houk JC (2003) Modulation of striatal single units by expected reward: a spiny neuron model displaying dopamine-induced bistability. J Neurophysiol 90:10951114. CrossRef Medline

Gurney KN, Prescott TJ, Redgrave P (2001a) A computational model of action selection in the basal ganglia. I. A new functional anatomy. Biol Cybern 84:401-410.

Gurney KN, Prescott TJ, Redgrave P (2001b) A computational model of action selection in the basal ganglia. II. Analysis and simulation of behaviour. Biol Cybern 84:411-423. CrossRef Medline

Halliday DM, Rosenberg JR, Amjad a M, Breeze P, Conway BA, Farmer SF (1995) A framework for the analysis of mixed time series/point process data-theory and application to the study of physiological tremor, single motor unit discharges and electromyograms. Prog Biophys Mol Biol 64:237-278. Medline

Hallworth NE, Wilson CJ, Bevan MD (2003) Apamin-sensitive small conductance calcium-activated potassium channels, through their selective coupling to voltage-gated calcium channels, are critical determinants of the precision, pace, and pattern of action potential generation in rat subthalamic nu. J Neurosci 23:7525-7542.

Hanson JE, Jaeger D (2002) Short-term plasticity shapes the response to simulated normal and parkinsonian input patterns in the globus pallidus. J Neurosci 22:5164-5172. Medline

Hernández A, Ibáñez-Sandoval O, Sierra A, Valdiosera R, Tapia D, Anaya V, Galarraga E, Bargas J, Aceves J (2006) Control of the subthalamic innervation of the rat globus pallidus by D2/3 and D4 dopamine receptors. J Neurophysiol 96:2877-2888. CrossRef Medline

Hernández-Echeagaray E, Starling AJ, Cepeda C, Levine MS (2004) Modulation of AMPA currents by D2 dopamine receptors in striatal medium-sized spiny neurons: are dendrites necessary? Eur J Neurosci 19:2455-2463. CrossRef Medline

Hornykiewicz O (1966) Dopamine (3-hydroxytyramine) and brain function. Pharmacol Rev. 18:925-964.

Houk JC, Beiser D (1998) Model of cortical-basal ganglionic processing: encoding the serial order of sensory events. J Neurophysiol 79:3168-3188. Medline

Humphries MD, Lepora N, Wood R, Gurney KN (2009a) Capturing dopaminergic modulation and bimodal membrane behaviour of striatal medium spiny neurons in accurate, reduced models. Front Comput Neurosci 3:26.

Humphries MD, Stewart RD, Gurney KN (2006) A physiologically plausible model of action selection and oscillatory activity in the basal ganglia. J Neurosci 26:12921-12942. CrossRef Medline

Humphries MD, Wood R, Gurney KN (2009b) Dopamine-modulated dynamic cell assemblies generated by the GABAergic striatal microcircuit. Neural Netw 22:1174-1188.

Ibañez-Sandoval O, Hernández A, Florán B, Galarraga E, Tapia D, Valdiosera R, Erlij D, Aceves J, Bargas J (2006) Control of the subthalamic innervation of substantia nigra pars reticulata by D1 and D2 dopamine receptors. J Neurophysiol 95:1800-1811. CrossRef Medline

Ingham CA, Hood SH, Mijnster MJ, Baldock R a., Arbuthnott GW (1997) Plasticity of striatopallidal terminals following unilateral le- sion of the dopaminergic nigrostriatal pathway: a morphological study. Exp Brain Res 116:39-49. Medline

Izhikevich EM (2007) Dynamical systems in neuroscience: The geometry of excitability and bursting. Cambridge, MA: MIT Press.

Izhikevich EM (2010) Hybrid spiking models. Philos Trans A Math Phys Eng Sci 368:5061-5070. CrossRef Medline

Jaeger D, Kita H (2011) Functional connectivity and integrative properties of globus pallidus neurons. Neuroscience 198:44-53. CrossRef Medline

Jaeger D, Kita H, Wilson CJ (1994) Surround inhibition among projection neurons is weak or nonexistent in the rat neostriatum. $J$ Neurophysiol 72:2555-2558. Medline

Kamali Sarvestani I, Lindahl M, Hellgren-Kotaleski J, Ekeberg Ö, Ekeberg O (2011) The arbitration-extension hypothesis: a hierarchical interpretation of the functional organization of the basal ganglia. Front Syst Neurosci 5:13. CrossRef Medline

Kincaid AE, Penney JB, Young AB, Newman SW (1991) The globus pallidus receives a projection from the parafascicular nucleus in the rat. Brain Res 553:18-26. Medline

Kita H, Kitai ST (1994) The morphology of globus pallidus projection neurons in the rat: an intracellular staining study. Brain Res 636: 308-319. Medline

Klaus A, Planert H, Hjorth JJJ, Berke JD, Silberberg G, Kotaleski JH (2011) Striatal fast-spiking interneurons: from firing patterns to postsynaptic impact. Front Syst Neurosci 5:57. CrossRef Medline

Koós T, Tepper JM (1999) Inhibitory control of neostriatal projection neurons by GABAergic interneurons. Nat Neurosci 2:467-472. CrossRef Medline

Koos T, Tepper JM, Wilson CJ (2004) Comparison of IPSCs evoked by spiny and fast-spiking neurons in the neostriatum. J Neurosci 24:7916-7922. CrossRef Medline

Kravitz AAV, Freeze BS, Parker PRLP, Kay K, Thwin MT, Deisseroth K, Kreitzer AC (2010) Regulation of parkinsonian motor behaviours by optogenetic control of basal ganglia circuitry. Nature 466:622626. CrossRef Medline

Kreiss DS, Mastropietro CW, Rawji SS, Walters JR (1997) The response of subthalamic nucleus neurons to dopamine receptor stimulation in a rodent model of Parkinson's disease. J Neurosci 17:6807-6819. Medline

Kreitzer AC, Malenka RC (2007) Endocannabinoid-mediated rescue of striatal LTD and motor deficits in Parkinson's disease models. Nature 445:643-647. CrossRef Medline

Kumar A, Cardanobile S, Rotter S, Aertsen A (2011) The role of inhibition in generating and controlling Parkinson's disease oscillations in the basal ganglia. Front Syst Neurosci 5:86. CrossRef Medline

Kumar A, Schrader S, Aertsen A, Rotter S (2008) The highconductance state of cortical networks. Neural Comput 20:1-43. CrossRef Medline

Levine MS, Li Z, Cepeda C, Cromwell HC, Altemus KL (1996) Neuromodulatory actions of dopamine on synaptically-evoked neostriatal responses in slices. Synapse 24:65-78. CrossRef Medline

Lindahl M, Kamali Sarvestani I, Ekeberg O, Kotaleski JH (2013) Signal enhancement in the output stage of the basal ganglia by synaptic short-term plasticity in the direct, indirect, and hyperdirect pathways. Front Comput Neurosci 7:76. CrossRef Medline

Magill PJ, Bolam JP, Bevan MD (2001) Dopamine regulates the impact of the cerebral cortex on the subthalamic nucleus-globus pallidus network. Neuroscience 106:313-330. Medline

Mallet N, Micklem BR, Henny P, Brown MT, Williams C, Bolam JP, Nakamura KC, Magill PJ (2012) Dichotomous organization of the external globus pallidus. Neuron 74:1075-1086. CrossRef Medline Mallet N, Pogosyan A, Márton LF, Bolam JP, Brown P, Magill PJ (2008) Parkinsonian beta oscillations in the external globus pallidus and their relationship with subthalamic nucleus activity. J Neurosci 28:14245-14258. CrossRef Medline

Mallet N, Schmidt R, Leventhal D, Chen F, Amer N, Boraud T, Berke JD (2016) Arkypallidal cells send a stop signal to striatum. Neuron 89:308-316. CrossRef Medline 
Markram H, Gupta A, Uziel A, Wang Y, Tsodyks M (1998) Information processing with frequency-dependent synaptic connections. Neurobiol Learn Mem 70:101-112. CrossRef Medline

Maurice N, Thierry A-M, Glowinski J, Deniau J-M (2003) Spontaneous and evoked activity of substantia nigra pars reticulata neurons during high-frequency stimulation of the subthalamic nucleus. $J$ Neurosci 23:9929-9936. Medline

McCairn KW, Bronfeld M, Belelovsky K, Bar-Gad I (2009) The neurophysiological correlates of motor tics following focal striatal disinhibition. Brain 132:2125-2138. CrossRef Medline

Miguelez C, Morin S, Martinez A, Goillandeau M, Bezard E, Bioulac B, Baufreton J (2012) Altered pallido-pallidal synaptic transmission leads to aberrant firing of globus pallidus neurons in a rat model of Parkinson's disease. J Physiol 590:5861-5875. CrossRef

Miller BR, Walker AG, Shah AS, Barton SJ, Rebec G V (2008) Dysregulated information processing by medium spiny neurons in striatum of freely behaving mouse models of Huntington's disease. J Neurophysiol 100:2205-2216. CrossRef Medline

Mink JW (1996) The basal ganglia: focused selection and inhibition of competing motor programs. Prog Neurobiol 50:381-425. Medline

Moyer JT, Wolf JA, Finkel LH (2007) Effects of dopaminergic modulation on the integrative properties of the ventral striatal medium spiny neuron. J Neurophysiol 98:3731-3748. CrossRef Medline

Nakanishi H, Kita H, Kitai ST (1987) Intracellular study of rat substantia nigra pars reticulata neurons in an in vitro slice preparation: electrical membrane properties and response characteristics to subthalamic stimulation. Brain Res 437:45-55. CrossRef

Nakanishi H, Kita H, Kitai ST (1991) Intracellular study of rat entopeduncular nucleus neurons in an in vitro slice preparation: response to subthalamic stimulation. Brain Res 549:285-291. CrossRef

Nakanishi H, Tamura A, Kawai K, Yamamoto K (1997) Electrophysiological studies of rat substantia nigra neurons in an in vitro slice preparation after middle cerebral artery occlusion. Neuroscience 77:1021-1028. CrossRef

Nambu A (2008) Seven problems on the basal ganglia. Curr Opin Neurobiol 18:595-604. CrossRef Medline

Nambu A, Llinaś R (1994) Electrophysiology of globus pallidus neurons in vitro. J Neurophysiol 72:1127-1139. Medline

Nevado-Holgado AJ, Mallet N, Magill PJ, Bogacz R (2014) Effective connectivity of the subthalamic nucleus-globus pallidus network during Parkinsonian oscillations. J Physiol 592:1429-1455. CrossRef Medline

Nisenbaum ES, Xu ZC, Wilson CJ (1994) Contribution of a slowly inactivating potassium current to the transition to firing of neostriatal spiny projection neurons. J Neurophysiol 71:1174-1189. Medline

Okun MS, Vitek JL (2004) Lesion therapy for Parkinson's disease and other movement disorders: update and controversies. Mov Disord 19:375-389. CrossRef Medline

Oorschot DE (1996) Total number of neurons in the neostriatal, pallidal, subthalamic, and substantia nigral nuclei of the rat basal ganglia: a stereological study using the cavalieri and optical disector methods. J Comp Neurol 366:580-599. CrossRef Medline

Park MR, Falls WM, Kitai ST (1982) An intracellular HRP study of the rat globus pallidus. I. Responses and light microscopic analysis. J Comp Neurol 211:284-294. CrossRef Medline

Planert H, Berger TK, Silberberg G (2013) Membrane properties of striatal direct and indirect pathway neurons in mouse and rat slices and their modulation by dopamine. PLoS One 8:e57054. CrossRef Medline

Planert H, Szydlowski SN, Hjorth J, Grillner S, Silberberg G (2010) Dynamics of synaptic transmission between fast-spiking interneurons and striatal projection neurons of the direct and indirect pathways. J Neurosci 30:3499-3507. CrossRef Medline

Potjans W, Diesmann M, Morrison A (2011) An imperfect dopaminergic error signal can drive temporal-difference learning. PLoS Comput Biol 7:e1001133. CrossRef Medline

Puccini GD, Sanchez-Vives M V, Compte A (2007) Integrated mechanisms of anticipation and rate-of-change computations in cortical circuits. PLoS Comput Biol 3:e82. CrossRef Medline
Rav-Acha M, Sagiv N, Segev I, Bergman H, Yarom Y (2005) Dynamic and spatial features of the inhibitory pallidal GABAergic synapses. Neuroscience 135:791-802. CrossRef Medline

Redgrave P, Prescott TJ, Gurney KN (1999) The basal ganglia: a vertebrate solution to the selection problem? Neuroscience 89: 1009-1023. Medline

Richards CD, Shiroyama T, Kitai ST (1997) Electrophysiological and immunocytochemical characterization of GABA and dopamine neurons in the substantia nigra of the rat. Neuroscience 80:545557. Medline

Sadek AR, Magill PJ, Bolam JP (2007) A single-cell analysis of intrinsic connectivity in the rat globus pallidus. J Neurosci 27: 6352-6362. CrossRef Medline

Saunders A, Huang KW, Sabatini BL (2016) Globus pallidus externus neurons expressing parvalbumin interconnect the subthalamic nucleus and striatal interneurons. PLoS One 11:e0149798. CrossRef Medline

Schmidt R, Leventhal DK, Mallet N, Chen F, Berke JD (2013) Canceling actions involves a race between basal ganglia pathways. Nat Neurosci 16:1118-1124. CrossRef Medline

Shen KZ, Johnson SW (2000) Presynaptic dopamine D2 and muscarine $\mathrm{M} 3$ receptors inhibit excitatory and inhibitory transmission to rat subthalamic neurones in vitro. J Physiol 525Pt2:331-341. Medline

Shen K-Z, Johnson SW (2006) Subthalamic stimulation evokes complex EPSCs in the rat substantia nigra pars reticulata in vitro. J Physiol 573:697-709. CrossRef Medline

Shen W, Flajolet M, Greengard P, Surmeier DJ (2008) Dichotomous dopaminergic control of striatal synaptic plasticity. Science 321: 848-851. CrossRef Medline

Sims RE, Woodhall GL, Wilson CL, Stanford IM (2008) Functional characterization of GABAergic pallidopallidal and striatopallidal synapses in the rat globus pallidus in vitro. Eur $\mathrm{J}$ Neurosci 28: 2401-2408. CrossRef Medline

Smith Y, Bevan MD, Shink E, Bolam JP (1998) Microcircuitry of the direct and indirect pathways of the basal ganglia. Neuroscience 86:353-387. Medline

Smith Y, Galvan A, Raju D, Wichmann T (2010) Anatomical and functional organization if thalamostriatal system. In: Handbook of Basal Ganglia Structure and Function (Steiner H, Tseng K, eds), pp 381-396. London: Elsevier.

Stewart TC, Bekolay T, Eliasmith C (2012) Learning to select actions with spiking neurons in the Basal Ganglia. Front Neurosci 6:2. CrossRef Medline

Taverna S, llijic E, Surmeier DJ (2008) Recurrent collateral connections of striatal medium spiny neurons are disrupted in models of Parkinson's disease. J Neurosci 28:5504-5512. CrossRef Medline Taverna S, van Dongen YC, Groenewegen HJ, Pennartz CMA (2004) Direct physiological evidence for synaptic connectivity between medium-sized spiny neurons in rat nucleus accumbens in situ. $J$ Neurophysiol 91:1111-1121. CrossRef

Tepper JM (2010) GABAergic interneurons of the striatum. In: Handbook of Basal Ganglia Structure and Function (Steiner H, Tseng $\mathrm{KY}$, eds), pp 151-165. London: Elsevier.

Tepper JM, Koós T, Wilson CJ (2004) GABAergic microcircuits in the neostriatum. Trends Neurosci 27:662-669. CrossRef Medline

Terman D, Rubin JE, Yew AC, Wilson CJ (2002) Activity patterns in a model for the subthalamopallidal network of the basal ganglia. $J$ Neurosci 22:2963-2976. Medline

Tsodyks M, Pawelzik K, Markram H (1998) Neural networks with dynamic synapses. Neural Comput 10:821-835. Medline

Tsodyks M V, Markram H (1997) The neural code between neocortical pyramidal neurons depends on neurotransmitter release probability. Proc Natl Acad Sci U S A 94:719-723. Medline

Tunstall MJ, Oorschot DE, Kean A, Wickens JR (2002) Inhibitory interactions between spiny projection neurons in the rat striatum. J Neurophysiol 88:1263-1269. Medline

Walters JR, Hu D, Itoga CA, Parr-Brownlie LC, Bergstrom DA (2007) Phase relationships support a role for coordinated activity in the indirect 
pathway in organizing slow oscillations in basal ganglia output after loss of dopamine. Neuroscience 144:762-776. CrossRef Medline

Wickens JR (1997) Basal ganglia: structure and computations. Netw Comput Neural Syst 8:R77-R109. CrossRef

Wickens JR, Arbuthnott GW, Shindou T (2007) Simulation of GABA function in the basal ganglia: computational models of GABAergic mechanisms in basal ganglia function. Prog Brain Res 160:313329. CrossRef Medline

Wilson CJ (1993) The generation of natural firing patterns in neostriatal neurons. Prog Brain Res 99:277-297. Medline
Yasukawa T, Kita T, Xue Y, Kita H (2004) Rat intralaminar thalamic nuclei projections to the globus pallidus: a biotinylated dextran amine anterograde tracing study. J Comp Neurol 471:153-167. CrossRef Medline

Zahr NM, Martin LP, Waszczak BL (2004) Subthalamic nucleus lesions alter basal and dopamine agonist stimulated electrophysiological output from the rat basal ganglia. Synapse 54:119-128. CrossRef Medline

Zhou F-W, Jin Y, Matta SG, Xu M, Zhou F-M (2009) An ultra-short dopamine pathway regulates basal ganglia output. J Neurosci 29:10424-10435. CrossRef Medline 